\title{
On the Asymptotic Convergence of Collocation Methods
}

\author{
By Douglas N. Arnold and Wolfgang L. Wendland*
}

\author{
This paper is dedicated to \\ Prof Dr.phil. Dr.h.c. Dr.h.c. Wolfgang Haack \\ on the occasion of his 80 th birthday
}

\begin{abstract}
We prove quasioptimal and optimal order estimates in various Sobolev norms for the approximation of linear strongly elliptic pseudodifferential equations in one independent variable by the method of nodal collocation by odd degree polynomial splines. The analysis pertains in particular to many of the boundary element methods used for numerical computation in engineering applications. Equations to which the analysis is applied include Fredholm integral equations of the second kind, certain first kind Fredholm equations, singular integral equations involving Cauchy kernels, a variety of integro-differential equations, and two-point boundary value problems for ordinary differential equations. The error analysis is based on an equivalence which we establish between the collocation methods and certain nonstandard Galerkin methods. We compare the collocation method with a standard Galerkin method using splines of the same degree, showing that the Galerkin method is quasioptimal in a Sobolev space of lower index and furnishes optimal order approximation for a range of Sobolev indices containing and extending below that for the collocation method, and so the standard Galerkin method achieves higher rates of convergence.
\end{abstract}

1. Introduction. In this paper we apply the method of nodal collocation by odd degree polynomial splines to systems of strongly elliptic pseudodifferential equations on closed curves and to two-point boundary value problems for ordinary differential equations. (By splines we always mean smoothest splines.) The former class of problems encompasses many of the boundary integral equations discretized by boundary element methods including some first kind and second kind Fredholm integral equations, singular integral equations with Cauchy kernels, and certain integro-differential equations such as the normal derivative of the double layer potential and the operator of Prandtl's wing theory.

For spline approximation of such strongly elliptic equations via standard Galerkin procedures or via the corresponding numerically integrated Galerkin-collocation methods the asymptotic error analysis is already rather completely developed; see

Received June 21, 1982.

1980 Mathematics Subject Classification. Primary 65R20; Secondary 65R99, 65L10, 65N99, 45J05, 45L10, 45F15, 35S99, 30C30, 73K30, 31A30.

* This work of Professor Arnold was supported in part by the National Science Foundation under contract MCS-81-02012. The research of Professor Wendland was supported in part by the Deutsche Forschungsgemeinschaft under project number We 659-04 and by the Department of Mathematics of the University of Maryland, College Park, and was partially carried out while he was a visitor to the University of Maryland Special Year in Numerical Analysis in 1981. 
[54], [55], [73], [79], [92], [102] and [50], [51], [100]. However, in practice most numerical computations based on boundary integral methods employ collocation procedures as in [7], [8], [20], [27], [56], [60], [68], [79], [99] and the proceedings [18], [24], [25], [26], [91]. Notwithstanding, few convergence results have yet been proved for spline collocation methods except in certain special cases. In the case of Fredholm integral equations of the second kind with smoothing integral operators, there is a large literature of which we refer only to the extensive bibliography of $\mathrm{B}$. Noble [75], the surveys of K. Atkinson [12] and C. Baker [17], and the results on superconvergence in [30], [42], [55], [82], [88]. Spline collocation of boundary value problems for ordinary differential equations has been studied by many authors and error estimates of various sorts have been proved in [9], [10], [21], [35], [66], [89]. There is also a sizeable literature on collocation of ordinary differential equations by less than smoothest splines [22], [36], [49], [66], [76], [87]. For the other types of equations considered here there are at present only preliminary results available for the special case of Fredholm integral equations of the first kind with logarithmic kernel [1], [2], [5], [98], and the recent work of S. Prössdorf and G. Schmidt on piecewise linear collocation for singular integral equations with Cauchy kernel [80], [81].

In this paper we introduce a new technique of proof and obtain convergence results in various spaces for splines of arbitrary odd degree and for a wide class of equations. To our knowledge, for the rather general class of strongly elliptic pseudodifferential equations considered, we present here the first rigorous convergence results for the collocation method. (The estimates announced in [101, (3.6), (3.7), (3.11)] are not correct and must be modified whereas those in [103, Chapter 9.7] are correct.)

It should be pointed out that the consistency of the methods, i.e., the convergence of the approximating operators on sufficiently smooth functions and the completeness of the trial functions as in [105, pp. 64-65], in general does not imply the convergence of the approximate solutions to the actual solution. L. Collatz has given a famous example of this phenomenon in [33, pp. 260-262]. Also the a posteriori error estimates in [7], [8] do not insure the convergence of the collocation method. Thus the question of convergence requires a more careful and thorough theoretical investigation.

The present convergence analysis is based on an equivalence between the collocation method and a nonstandard Galerkin method for an operator closely related to the given operator. The Galerkin method is nonstandard in that the pairing of test and trial functions is made in the inner product of the Sobolev space of order $(d+1) / 2$ where $d$ denotes the degree of the splines, rather than in the $L^{2}$ inner product like the standard Galerkin method. Once the equivalence is shown, the procedure is analyzed by well-established techniques. We show that quasioptimal approximation is achieved in a Sobolev space with index depending on the order of the equation and the degree of the splines. We also establish optimal order estimates in a range of Sobolev spaces. This range is strictly limited, as we show that there is a maximal order of convergence which cannot be exceeded in any Sobolev space. A similar situation obtains for the standard Galerkin method, and a comparison of the two methods shows that to obtain the same maximal order of convergence as the mesh size decreases, one must choose the degree of the splines for the collocation 
method one greater than twice the degree used in the Galerkin method. From this point of view, collocation appears to be less efficient. On the other hand the collocation method requires only that one integration be performed for each element of the stiffness matrix, whereas the standard Galerkin method requires double integration, and hence an economic implementation of the Galerkin method requires an efficient scheme for evaluation of the matrix (as, e.g., in the Galerkin-collocation methods [50], [51], [100]).

The paper is divided into two major parts. In Section 2 we consider the periodic case encompassing the boundary element methods. There we proved quasioptimal and optimal order error estimates for the case of single equations, and the estimates are shown to be sharp. Then we compare the results with the standard Galerkin method. We also estimate the conditioning of the discrete equations. Next we generalize the estimates to the case of certain systems of equations of a form which arises frequently in applications, e.g., in Fichera's method with Fredholm integral equations of the first kind [37], [50], [51], [52], [53]. We then verify that strongly elliptic pseudodifferential operators satisfy all the assumptions made in the analysis. As applications we present elliptic integro-differential equations and consider the special cases of singular integral equations, Fredholm integral equations of the second kind, the normal derivative of the double layer potential, and Prandtl's wing operator. Another type of equation, the Fredholm integral equation of the first kind with logarithmic kernel, is also discussed in detail. Applications of such equations to a wide variety of physical problems are reviewed.

In Section 3 we indicate the application of our methods to nonperiodic problems by considering one example, that of cubic spline collocation of a second order two-point Dirichlet problem. Error estimates similar to those of Section 2 are established. This section is presented in an elementary fashion and may be read independently of Section 2.

In an Appendix we present a construction from [81] which furnishes the matrix transforming a strongly elliptic problem into a coercive one, and then we formulate the singular integral equations of the direct method for plane elasticity.

Although this analysis provides error estimates for a large class of collocation methods which occur in applications, there remain a number of important collocation methods to which our results do not apply. In particular we cannot yet handle the case of splines of even degree, nor methods violating the conformity assumption that the degree of the splines exceeds the order of the operator. Our work does not pertain to systems of equations of differing orders, and further considerations are needed to apply our method to integral equations arising from boundary value problems on corner domains or mixed boundary value problems. Also we do not consider collocating functions other than smoothest splines, nor collocation at points other than the nodes.

2. Collocation of Pseudodifferential Equations on Closed Curves. Let $\Gamma$ be a plane Jordan curve given by a regular parametric representation

$$
\Gamma: z=\left(z_{1}(t), z_{2}(t)\right) \cong z_{1}(t)+i z_{2}(t),
$$

where $z$ is a l-periodic function of a real variable and $|d z / d t| \neq 0$. Via the parametrization we have a one-to-one correspondence between functions on $\Gamma$ and 
1-periodic functions. More generally, for a system of mutually disjoint Jordan curves $\Gamma=\cup_{j=1}^{L} \Gamma_{j}$ we may parametrize each and identify functions on $\Gamma$ with $L$-vector valued 1-periodic functions. We thus limit ourselves without loss of generality to systems of equations of the form

$$
A u+B \omega=f, \quad \Lambda u=\beta,
$$

where the 1-periodic vector valued function $u=\left(u_{1}(t), \ldots, u_{p}(t)\right)$ and the vector $\omega \in \mathbf{R}^{q}$ denote the desired unknowns, $f=\left(f_{1}(t), \ldots, f_{p}(t)\right)$ and $\beta \in \mathbf{R}^{q}$ a given function and vector, $A$ a given linear operator, $B$ a given matrix of functions and $\Lambda$ a given vector valued functional.

For simplicity we consider first the special case of (2.1) of just one equation ( $p=1)$ and for which $B, \Lambda$, and $\beta$ vanish.

2.1. Collocation of a Single Equation. Let $A$ be a given bounded linear mapping $H^{j+\alpha} \rightarrow H^{j-\alpha}$ with $j \in \mathbf{R}$ to be specified later. The quantity $2 \alpha$ is called the order of $A$. Here $H^{s}$ denotes the periodic Sobolev space of arbitrary real order $s$, i.e., the closure of all smooth real-valued 1-periodic functions with respect to the norm

$$
\|f\|_{s}:=\|f\|_{H^{s}}:=\left\{\left|\hat{f}_{0}\right|^{2}+\sum_{0 \neq k \in \mathbf{Z}}\left|\hat{f}_{k}\right|^{2}|2 \pi k|^{2 s}\right\}^{1 / 2},
$$

where

$$
\hat{f}_{k}=\int_{0}^{1} e^{-2 \pi i k t} f(t) d t, \quad k \in \mathbf{Z},
$$

are the Fourier coefficients. We denote the inner product in this space by

$$
\langle f, g\rangle_{s}:=\hat{f}_{0} \cdot \overline{\hat{g}}_{0}+\sum_{0 \neq k \in \mathbf{Z}} \hat{f}_{k} \cdot \overline{\hat{g}}_{k}|2 \pi k|^{2 s} .
$$

Note that this inner product extends to a duality pairing between $H^{s+\alpha}$ and $H^{s-\alpha}$ for arbitrary real $\alpha$, and moreover

$$
\sup _{w \in H^{s-\alpha}} \frac{\langle v, w\rangle_{s}}{\|w\|_{s-\alpha}}=\|v\|_{s+\alpha}, \quad v \in H^{s+\alpha} .
$$

Clearly $H^{s}$ is continuously imbedded in $H^{t}$ for $s>t$.

The aim of this section is to investigate the approximation of the equation

$$
A u=f
$$

by the method of collocation. To this end we select an increasing sequence of mesh points $\Delta=\left\{x_{i}\right\}_{i=-\infty}^{\infty}$ satisfying $x_{i+n}=x_{i}+1$, for fixed $n \in \mathbf{N}$ and all $i \in \mathbf{Z}$, and denote by $S_{d}(\Delta)$ the space of all 1-periodic, $d-1$ times continuously differentiable splines of degree $d$ subordinate to the partition $\Delta$. Note that, since $\phi^{(d)}$ (the superscript denotes differentiation) is a step function for $\phi \in S_{d}(\Delta), S_{d}(\Delta) \subset H^{s}$ if and only if $s<d+\frac{1}{2}$. We require that the degree of the splines be odd and henceforth denote by $d$ a fixed positive odd integer and by $j$ the integer $(d+1) / 2$.

Let $h_{\Delta}=\max \left(x_{i}-x_{i-1}\right)$. We recall the approximation proeprties of $S_{d}(\Delta)$.

If $-\infty<t \leqslant s \leqslant d+1$ and $t<d+\frac{1}{2}$, then there exists a constant $C$ depending only on $t, s$, and $d$ such that

$$
\inf _{\phi \in S_{d}(\Delta)}\|u-\phi\|_{t} \leqslant C h_{\Delta}^{s-t}\|u\|_{s}, \quad u \in H^{s}
$$


(Throughout, $C$ denotes a generic constant independent of $\Delta$, not the same at each occurrence.) This result is proven in many places under the additional assumption that $0 \leqslant t \leqslant d$, e.g., [89, Chapter 6], [14], [104]. In [15, Theorem 4.1.2] and [46] the restriction that $t$ (and therefore $s$ ) be nonnegative is removed. Helfrich showed in [46] even the following stronger approximation property for $t \leqslant d$ and $t \leqslant s$ :

To any $u \in H^{s}$ and $S_{d}(\Delta)$ there exists $\psi \in S_{d}(\Delta)$ such that

$$
\|u-\psi\|_{t} \leqslant C h_{\Delta}^{s-t}\|u\|_{s}
$$

where $\psi$ is independent of $t$. However, the case $d<t<d+\frac{1}{2}$ does not appear to be in the literature. In the case of a uniform mesh the result can be proved quite easily using the Fourier series definition of the Sobolev norms (2.1.1) and using a $B$-spline basis for $S_{d}(\Delta)$. (Since every basis element can be realized as the translate of the iterated convolution of the characteristic function of an interval, the Fourier coefficients of the splines are easily found.) In the manuscript "The regularity of piecewise defined functions with resepct to scales of Sobolev spaces" (Corollary to Theorem 3), J. A. Nitsche has proved a result of this sort for approximation by piecewise constant functions subordinate to a general regular triangulation of a plane domain. For the analogous result in one dimension one can derive the result by using the $d$ th derivative operator to map $S_{d}(\Delta)$ into $S_{0}(\Delta)$.

The collocation method for (2.1.3) reads as: Find $u_{\Delta} \in S_{d}(\Delta)$ such that the collocation equations

$$
\left(A u_{\Delta}\right)\left(x_{i}\right)=f\left(x_{i}\right), \quad i=1, \ldots, n,
$$

are satisfied.

Since $A u_{\Delta} \in H^{s-2 \alpha}$ only for $s<d+\frac{1}{2}$, and since $A u_{\Delta}$ must be continuous at the nodes in order that we may collocate, we are led to the assumption that for some $s<d+\frac{1}{2}$ the space $H^{s-2 \alpha}$ is embedded in the space of continuous functions. In view of the Sobolev imbedding theorem we thus assume the relation

$$
d=2 j-1>2 \alpha \text { or, equivalently, } j-\alpha>\frac{1}{2}
$$

between the order of the operator $A$ and the degree of the splines. This assumption impies in particular the inclusion

$$
S_{d}(\Delta) \subset H^{j+\alpha} \text {. }
$$

We require in addition the following properies of $A$.

$$
A: H^{j+\alpha} \rightarrow H^{j-\alpha} \text { is an isomorphism, }
$$

i.e., $A$ is bijective and so Eq. (2.1.3) is uniquely solvable.

$$
A \text { is j-coercive, i.e., A satisfies the Gårding inequality }
$$

$$
\langle A u, u\rangle_{j} \geqslant \gamma\|u\|_{j+\alpha}^{2}-\langle K u, u\rangle_{j}, \quad u \in H^{j+\alpha},
$$

where $\gamma>0$ and $K$ is a compact linear operator $H^{j+\alpha} \rightarrow H^{j-\alpha}$.

Note that (A.3) holds if there exist constants $\gamma, \varepsilon>0, \gamma^{\prime} \in \mathbf{R}$ such that

$$
\langle A u, u\rangle_{j} \geqslant \gamma\|u\|_{j+\alpha}^{2}-\gamma^{\prime}\|u\|_{j+\alpha-\varepsilon}^{2} .
$$

For any bounded linear operator $B: H^{s} \rightarrow H^{t}$ we define its formal $j$-adjoint $B^{*}$ : $H^{2 j-t} \rightarrow H^{2 j-s}$ by

$$
\left\langle u, B^{*} v\right\rangle_{j}=\langle B u, v\rangle_{j} \text { for all } u \in H^{s}, v \in H^{2 j-t} .
$$


Obviously $A^{*}$ also satisfies the coercivity inequality (2.1.6). This together with assumption (A.2) implies that $A$ and $A^{*}$ are both Fredholm mappings of index zero and that $A^{*}$ is also an isomorphism from $H^{j+\alpha}$ onto $H^{j-\alpha}$ [93]. Therefore both mappings satisfy a priori estimates of the form

$$
\|u\|_{j+\alpha} \leqslant C\|A u\|_{j-\alpha} \text { and }\|u\|_{j+\alpha} \leqslant C\left\|A^{*} u\right\|_{j-\alpha}
$$

i.e., ellipticity.

We now reduce the collocation equations (2.1.5) to equivalent Galerkin equations. To this end we define mappings $J$ and $J_{\Delta}$ by the integral and its numerical counterpart, the trapezoidal rule, namely

$$
J u:=\int_{0}^{1} u(x) d x \text { and } J_{\Delta} u:=\sum_{i=1}^{n} \delta_{i} u\left(x_{i}\right), \quad \text { where } \delta_{i}:=\left(x_{i+1}-x_{i-1}\right) / 2 \text {. }
$$

THEOREM 2.1.1. Let $w \in H^{j-\alpha}$. Then the equations

$$
w\left(x_{i}\right)=0, \quad i=1, \ldots, n,
$$

hold if and only if

$$
\left\langle w-J w+J_{\Delta} w, v\right\rangle_{j}=0 \quad \text { for all } v \in S_{d}(\Delta) .
$$

Note that by assumption (A.1) the point values referenced in (2.1.7) and (2.1.8) are well defined. Moreover the inclusion $S_{d}(\Delta) \subset H^{j+\alpha}$ insures that the inner product in (2.1.8) is defined.

Proof. Observe that

$$
\langle f, g\rangle_{j}=\int_{0}^{1} f^{(j)}(x) g^{(j)}(x) d x+\int_{0}^{1} f(x) d x \int_{0}^{1} g(x) d x
$$

for $f, g \in H^{j}$. Integrating by parts we find that

$$
\left\langle w-J w+J_{\Delta} w, v\right\rangle_{j}=(-1)^{j-1} \int_{0}^{1} w^{\prime}(x) v^{(2 j-1)}(x) d x+J_{\Delta} w \cdot J v .
$$

Now the operator $(d / d x)^{2 j-1}$ maps $\left\{v \in S_{d}(\Delta) \mid J v=0\right\}$ isomorphically onto $\{v \in$ $\left.S_{0}(\Delta) \mid J v=0\right\}$, the space of piecewise constant functions with integral zero. Define $y_{i}$ in this space by

$$
y_{i}(x)= \begin{cases}-h_{i}^{-1} & \text { for } x \in\left[x_{i-1}, x_{i}\right) \\ h_{i+1}^{-1} & \text { for } x \in\left[x_{i}, x_{i+1}\right) \\ 0 & \text { for } x \in\left[x_{i+1}, x_{i-1}+1\right)\end{cases}
$$

where $h_{i}=x_{i}-x_{i-1}$, and determine $v_{i} \in S_{d}(\Delta)$ by $v_{i}^{(2 j-1)}=y_{i}, J v_{i}=0$. Then

$$
\left\langle w-J w+J_{\Delta} w, v_{i}\right\rangle=(-1)^{j-1}\left[h_{i+1}^{-1}\left(w\left(x_{i+1}\right)-w\left(x_{i}\right)\right)-h_{i}^{-1}\left(w\left(x_{i}\right)-w\left(x_{i-1}\right)\right)\right] .
$$

Hence if (2.1.8) holds then there exists a constant $\kappa$ such that

$$
h_{i}^{-1}\left(w\left(x_{i}\right)-w\left(x_{i-1}\right)\right)=\kappa, \quad i \in \mathbf{Z} .
$$

Applying (2.1.9) repeatedly with $i=n, n-1, \ldots, 1$, we find that

$$
w\left(x_{n}\right)=w\left(x_{0}\right)+\kappa\left(h_{n}+h_{n-1}+\cdots+h_{1}\right)=w\left(x_{0}\right)+\kappa .
$$

Since $w$ is periodic, necessarily $\kappa=0$. Thus (2.1.8) implies

$$
w\left(x_{i}\right)=w\left(x_{n}\right) \text { for all } i \in \mathbf{Z} \text {. }
$$


In fact, since $S_{d}(\Delta)$ is spanned by the $v_{i}$ and the constant function 1 , we see that (2.1.8) holds if and only if (2.1.10) holds and, in addition,

$$
J_{\Delta} w=\left\langle w-J w+J_{\Delta} w, 1\right\rangle_{j}=0 .
$$

But clearly (2.1.10) and (2.1.11) are together equivalent to (2.1.7), and so the proof is complete.

We are now in a position to reformulate the collocation equations (2.1.5) as Galerkin equations. For this purpose we define the operator

$$
A_{\Delta}:=\left(I-J+J_{\Delta}\right) A: H^{j+\alpha} \rightarrow H^{j-\alpha}
$$

( $I$ denotes the identity) which is continuous since $j-\alpha>\frac{1}{2}$.

TheOREM 2.1.2. A function $u_{\Delta} \in S_{d}(\Delta)$ satisfies the collocation equations (2.1.5) if and only if it satisfies the Galerkin equations

$$
\left\langle A_{\Delta} u_{\Delta}, v\right\rangle_{j}=\left\langle A_{\Delta} u, v\right\rangle_{j}, \quad v \in S_{d}(\Delta),
$$

where $u$ is the solution of (2.1.3).

We proceed now to the proof that the Galerkin procedure (2.1.13) is uniformly bounded and stable, establishing first the uniform bijectivity of the operator $A_{\Delta}$.

LEMma 2.1.3. The operator $A_{\Delta}$ is invertible with inverse

$$
A_{\Delta}^{-1}=A^{-1}\left(I+J-J_{\Delta}\right) .
$$

Moreover there exists a constant $C$ such that

$$
\left\|A_{\Delta}\right\|_{j+\alpha, j-\alpha}+\left\|A_{\Delta}^{-1}\right\|_{j-\alpha, j+\alpha} \leqslant C
$$

for all partitions $\Delta$.

Proof. Since $j-\alpha>\frac{1}{2}, J_{\Delta}: H^{j-\alpha} \rightarrow H^{j-\alpha}$ is uniformly bounded, and so the uniform boundedness of $A_{\Delta}$ follows from the continuity of $A$. Equation (2.1.14) follows immediately from the identities

$$
J J=J_{\Delta} J=J \text { and } J_{\Delta} J_{\Delta}=J J_{\Delta}=J_{\Delta},
$$

and then the uniform boundedness of $A_{\Delta}^{-1}$ follows from the continuity of $A^{-1}$.

The following theorem asserts the stability of the Galerkin procedure (2.1.13).

THEOREM 2.1.4. There exist positive constants $C$ and $h_{0}$ such that if $\Delta$ is a partition for which $h_{\Delta}:=\max \left(x_{i}-x_{i-1}\right) \leqslant h_{0}$, then

$$
\inf _{\substack{v \in S_{d}(\Delta) \\\|v\|_{j+\alpha}=1}} \sup _{\substack{z \in S_{d}(\Delta) \\\|z\|_{j+\alpha}=1}}\left\langle A_{\Delta} v, z\right\rangle_{j} \geqslant C .
$$

Proof. From the assumption (A.1)-(A.3) we obtain with integration by parts the coerciveness inequality

$$
\left\langle(-1)^{j}\left(\frac{d}{d s}\right)^{2 j} A u+J A u, u\right\rangle_{0}=\langle A u, u\rangle_{j} \geqslant \gamma_{1}\|u\|_{j+\alpha}^{2}-k_{1}[u, u],
$$

where

$$
k_{1}[u, v]=\langle K u, v\rangle_{j}
$$


is a compact bilinear form on $H^{j+\alpha} \times H^{j+\alpha}$. Thus the results in [6], [28], [47], [55], [92] imply that the solution $w_{\Delta} \in S_{d}(\Delta)$ of

$$
\left\langle A w_{\Delta}, v_{\Delta}\right\rangle_{j}=\left\langle A w, v_{\Delta}\right\rangle_{j} \text { for all } v_{\Delta} \in S_{d}(\Delta)
$$

satisfies the estimate

$$
\left\|w_{\Delta}\right\|_{j+\alpha} \leqslant c\|w\|_{j+\alpha}
$$

for all $0<h_{\Delta} \leqslant h_{0}$ with an appropriate $h_{0}>0$. Then we reformulate the equation (2.1.13) for $u_{\Delta}$,

$$
\left\langle A_{\Delta} u_{\Delta}, v_{\Delta}\right\rangle_{j}=\left\langle A_{\Delta} u, v_{\Delta}\right\rangle_{j} \text { for all } v_{\Delta} \in S_{d}(\Delta)
$$

in the form

$$
\left\langle A u_{\Delta}, v_{\Delta}\right\rangle_{j}=\left\langle A\left(u-A^{-1}\left\{\left(J_{\Delta}-J\right) A u_{\Delta}-\left(J_{\Delta}-J\right) A u\right\}\right), v_{\Delta}\right\rangle_{j}
$$

which yields with (2.1.18) the estimate

$$
\begin{aligned}
\left\|u_{\Delta}\right\|_{j+\alpha} & \leqslant c\left(\|u\|_{j+\alpha}+\left\|A^{-1}\left(J_{\Delta}-J\right) A u_{\Delta}\right\|_{j+\alpha}+\left\|A^{-1}\left(J_{\Delta}-J\right) A u\right\|_{j+\alpha}\right) \\
& \leqslant c\left(\|u\|_{j+\alpha}+c_{1}\left(\left|\left(J_{\Delta}-J\right) A u_{\Delta}\right|+\left|\left(J_{\Delta}-J\right) A u\right|\right)\right) \\
& \leqslant c\left(\|u\|_{j+\alpha}+c_{2} h_{\Delta}^{\mu}\left(\left\|A u_{\Delta}\right\|_{j-\alpha}+\|A u\|_{j-\alpha}\right)\right) \\
& \leqslant c\|u\|_{j+\alpha}+c_{3} h_{\Delta}^{\mu}\left\|u_{\Delta}\right\|_{j+\alpha}+c_{3} h_{\Delta}^{\mu}\|u\|_{j+\alpha} .
\end{aligned}
$$

Here $\mu=\min (2, j-\alpha)>0$. Hence, for $0<h_{\Delta} \leqslant h_{0}$ and $c_{3} h_{\Delta}^{\mu} \leqslant \frac{1}{2}$ we obtain the stability estimate

$$
\left\|u_{\Delta}\right\|_{j+\alpha} \leqslant 3 c\|u\|_{j+\alpha}
$$

uniformly for all such $h_{\Delta}$. This in turn yields (2.1.15).

Remark. The authors are grateful to Dr. G. Schmidt and Professor Dr. S. Prössdorf for pointing out that Theorem 2.1.4 holds without an additional regularity assumption which appeared in an earlier draft.

In view of Theorem 2.1.2, Lemma 2.1.3, and Theorem 2.1.4 we may apply the standard theory of Galerkin methods [15], [16], [29] to show that the collocation procedure furnishes quasioptimal approximation in its natural energy space $H^{j+\alpha}$. Using the approximation property (2.1.4),we obtain further asymptotic error bounds.

THEOREM 2.1.5. There exist positive constants $C$ and $h_{0}$ such that for any partition $\Delta$ with $h_{\Delta} \leqslant h_{0}$ there is a unique solution $u_{\Delta} \in S_{d}(\Delta)$ of the collocation equations (2.1.5). Moreover we have quasioptimality in the form of Cea's lemma:

$$
\left\|u-u_{\Delta}\right\|_{j+\alpha} \leqslant C \inf _{v \in S_{d}(\Delta)}\|u-v\|_{j+\alpha} .
$$

If in addition the solution $u \in H^{s}$ for some $s \in[j+\alpha, 2 j]$, then

$$
\left\|u-u_{\Delta}\right\|_{j+\alpha} \leqslant C h_{\Delta}^{s-j-\alpha}\|u\|_{s} .
$$

Theorem 2.1.5 gives estimates for the collocation method in the space $H^{j+\alpha}$. We now extend these estimates to spaces of lower order by applying the Aubin-Nitsche duality argument. For $s>j+\alpha$ we say that an isomorphism $B: H^{j+\alpha} \rightarrow H^{j-\alpha}$ is $s$-regular if $B^{-1}$ maps $H^{s-2 \alpha}$ boundedly into $H^{s}$. 
THEOREM 2.1.6. Let $t \in[2 \alpha, j+\alpha]$, and assume that $A^{*}$ is $(2(j+\alpha)-t)$-regular. In case $t \leqslant 2 \alpha+\frac{1}{2}$ and $j>\alpha+2$, assume in addition that $A$ maps $H^{2+2 \alpha}$ boundedly into $H^{2}$. Then

$$
\left\|u-u_{\Delta}\right\|_{t} \leqslant C h_{\Delta}^{j+\alpha-t}\left\|u-u_{\Delta}\right\|_{j+\alpha} .
$$

If in addition $u \in H^{s}$ for some $s \in[j+\alpha, 2 j]$, then

$$
\left\|u-u_{\Delta}\right\|_{t} \leqslant C h_{\Delta}^{s-t}\|u\|_{s} .
$$

Proof. In light of Theorem 2.1.5, it suffices to demonstrate (2.1.21).

We first consider the case $t \in\left(2 \alpha+\frac{1}{2}, j+\alpha\right]$. (This interval is nonempty by (A.1).) We then have $2(j+\alpha)-t<2 j-\frac{1}{2}$, so the $j$-adjoint operators

$$
J_{\Delta}^{*}: H^{2(j+\alpha)-t} \rightarrow H^{2(j+\alpha)-t}
$$

are uniformly bounded. From the regularity assumption on $A^{*}$ it then follows that

$$
A_{\Delta}^{*-1}=\left(I+J-J_{\Delta}^{*}\right) A^{*-1}: H^{2 j-t} \rightarrow H^{2(j+\alpha)-t}
$$

is uniformly bounded.

Now there exists $v \in H^{2 j-t}$ such that

$$
\left\langle u-u_{\Delta}, v\right\rangle_{j}=\left\|u-u_{\Delta}\right\|_{t}^{2}=\|v\|_{2 j-t}^{2}
$$

by duality. Set $y=A_{\Delta}^{*-1} v$. Then the uniform boundedness of $A_{\Delta}^{*-1}$ and (2.1.23) imply

$$
\|y\|_{2(j+\alpha)-t} \leqslant C\|v\|_{2 j-t}=C\left\|u-u_{\Delta}\right\|_{t},
$$

and using (2.1.13) and (2.1.23) we find that

$$
\begin{aligned}
\left\|u-u_{\Delta}\right\|_{t}^{2} & =\left\langle\left(u-u_{\Delta}\right), A_{\Delta}^{*} y\right\rangle_{j}=\left\langle A_{\Delta}\left(u-u_{\Delta}\right), y\right\rangle_{j} \\
& =\inf _{\varphi \in S_{d}(\Delta)}\left\langle A_{\Delta}\left(u-u_{\Delta}\right), y-\varphi\right\rangle_{j} \\
& \leqslant C\left\|A_{\Delta}\left(u-u_{\Delta}\right)\right\|_{j-\alpha} \cdot \inf _{\varphi \in S_{d}(\Delta)}\|y-\varphi\|_{j+\alpha} \\
& \leqslant C\left\|u-u_{\Delta}\right\|_{j+\alpha} \cdot h_{\Delta}^{j+\alpha-t}\|y\|_{2(j+\alpha)-t} .
\end{aligned}
$$

Now the estimate (2.1.21) follows from (2.1.24) and (2.1.25).

We turn now to the remaining case of $t \in\left[2 \alpha, 2 \alpha+\frac{1}{2}\right]$. Again we define $v$ by (2.1.23). However, since in this case $A_{\Delta}^{*}$ is not $(2(j+\alpha)-t)$-regular, we set $y=A^{*-1} v$. Applying (2.1.23), we get

$$
\begin{aligned}
\left\|u-u_{\Delta}\right\|_{t}^{2} & =\left\langle\left(u-u_{\Delta}\right), A^{*} y\right\rangle_{j} \\
& =\left\langle A_{\Delta}\left(u-u_{\Delta}\right), y\right\rangle_{j}+\left\langle\left(A-A_{\Delta}\right)\left(u-u_{\Delta}\right), y\right\rangle_{j} .
\end{aligned}
$$

The first term on the right-hand side of (2.1.26) may be estimated as in (2.1.25). For the second term we have

$$
\begin{gathered}
\left|\left\langle\left(A-A_{\Delta}\right)\left(u-u_{\Delta}\right), y\right\rangle_{j}\right|=\left|\left(J-J_{\Delta}\right) A\left(u-u_{\Delta}\right)\right||J y| \\
\leqslant C\left|\left(J-J_{\Delta}\right) A\left(u-u_{\Delta}\right)\right|\|y\|_{2(j+\alpha)-t} \\
\quad \leqslant C\left|\left(J-J_{\Delta}\right) A\left(u-u_{\Delta}\right)\right|\left\|u-u_{\Delta}\right\|_{t} .
\end{gathered}
$$


If $j-\alpha \leqslant 2$ we use (A.1) and simple error estimates for the trapezoidal rule to see that

$$
\begin{aligned}
\left|\left(J-J_{\Delta}\right) A\left(u-u_{\Delta}\right)\right| & \leqslant C h_{\Delta}^{j-\alpha}\left\|A\left(u-u_{\Delta}\right)\right\|_{j-\alpha} \leqslant C h_{\Delta}^{j-\alpha}\left\|u-u_{\Delta}\right\|_{j+\alpha} \\
& \leqslant C h_{\Delta}^{j+\alpha-t}\left\|u-u_{\Delta}\right\|_{j+\alpha}
\end{aligned}
$$

and substitute these estimates in (2.1.26) to get (2.1.21). If $j-\alpha>2$, we invoke the additional boundedness assumptions to get

$$
\left|\left(J-J_{\Delta}\right) A\left(u-u_{\Delta}\right)\right| \leqslant c h_{\Delta}^{2}\left\|A\left(u-u_{\Delta}\right)\right\|_{2} \leqslant c h_{\Delta}^{2}\left\|u-u_{\Delta}\right\|_{2 \alpha+2} .
$$

Since $2 \alpha+2 \in\left(2 \alpha+\frac{1}{2}, j+\alpha\right)$, we may apply (2.1.21) with $t$ replaced by $2 \alpha+2$ by the previous case, yielding

$$
\left|\left(J-J_{\Delta}\right) A\left(u-u_{\Delta}\right)\right| \leqslant C h_{\Delta}^{j-\alpha}\left\|u-u_{\Delta}\right\|_{j+\alpha} \leqslant C h_{\Delta}^{j+\alpha-t}\left\|u-u_{\Delta}\right\|_{j+\alpha} .
$$

Finally, combining (2.1.27) and (2.1.28) and substituting into (2.1.26), we get (2.1.21).

Remark. For $t \in\left(2 \alpha, 2 \alpha+\frac{1}{2}\right]$ the last inequality in (2.1.28) is clearly not sharp and a careful analysis shows that the additional boundedness assumption can be slightly weakened in this case.

Remark. The highest order estimate which can be inferred from Theorems 2.1.5 and 2.1 .6 is

$$
\left\|u-u_{\Delta}\right\|_{2 \alpha}=O\left(h_{\Delta}^{2(j-\alpha)}\right),
$$

which holds for sufficiently smooth $u$. Note that this estimate is of precisely twice the order of the best approximation in the energy space $H^{j+\alpha}$. In fact no higher order estimate is possible, as is shown by the following argument which is based on an argument of Bramble and Scott [23] and applies quite generally to Galerkin methods.

For simplicity we assume that $A$ is strictly coercive (i.e., $K=0$ in (2.1.6)) and maps smooth functions onto smooth functions, and moreover that the true solution $u$ is smooth and has integral zero. Using (2.1.6), (2.1.12) and (2.1.13) we find that

$$
\begin{aligned}
\gamma \| u- & u_{\Delta} \|_{j+\alpha}^{2} \leqslant\left\langle A\left(u-u_{\Delta}\right), u-u_{\Delta}\right\rangle_{j} \\
& =\left\langle A_{\Delta}\left(u-u_{\Delta}\right), u-u_{\Delta}\right\rangle_{j}+\left\langle\left(J-J_{\Delta}\right) A\left(u-u_{\Delta}\right), u-u_{\Delta}\right\rangle_{j} \\
& =\left\langle A_{\Delta}\left(u-u_{\Delta}\right), u\right\rangle_{j}+\left(J-J_{\Delta}\right) A\left(u-u_{\Delta}\right) \cdot J\left(u-u_{\Delta}\right) .
\end{aligned}
$$

Now, since we assume that $J u=0$, we have for any $t \in \mathbf{R}$ that

$$
\left|\left\langle A_{\Delta}\left(u-u_{\Delta}\right), u\right\rangle_{j}\right|=\left|\left\langle A\left(u-u_{\Delta}\right), u\right\rangle_{j}\right| \leqslant C\left\|u-u_{\Delta}\right\|_{t}\left\|A^{*} u\right\|_{2 j-t} .
$$

Moreover $\left|J\left(u-u_{\Delta}\right)\right| \leqslant\left\|u-u_{\Delta}\right\|_{t}$, and, since $j-\alpha>\frac{1}{2}$,

$$
\left|\left(J-J_{\Delta}\right) A\left(u-u_{\Delta}\right)\right| \leqslant\left\|A\left(u-u_{\Delta}\right)\right\|_{j-\alpha} \leqslant C\left\|u-u_{\Delta}\right\|_{j+\alpha} \leqslant C\|u\|_{j+\alpha} .
$$

Substituting these bounds in (2.1.29), we see that

$$
\left\|u-u_{\Delta}\right\|_{j+\alpha}^{2} \leqslant M\left\|u-u_{\Delta}\right\|_{t},
$$

where the constant $M$ depends on $u$, but not on $\Delta$. Thus we see that the order of approximation of $u$ by $u_{\Delta}$ in an arbitrary Sobolev space $H^{t}$ cannot exceed twice its order in the space $H^{j+\alpha}$. For generic $u$, therefore, the best possible order is $O\left(h_{\Delta}^{2(j-\alpha)}\right)$. 
Having proved optimal order error bounds in $H^{t}$ for $t=j+\alpha$ and, when possible, for $t<j+\alpha$, we turn now to the case $t>j+\alpha$. For this case we shall require the inverse properties of spline spaces and hence must restrict ourselves to a quasiuniform family of meshes. A mesh $\Delta$ is said to be $\rho$-quasiuniform $(\rho>0)$ if $h_{\Delta} \leqslant \rho \inf _{i}\left(x_{i}-x_{i-1}\right)$.

The inverse properties state that for any real numbers $\rho, \sigma$, and $\tau$ satisfying $\rho>0$ and $\tau \leqslant \sigma<d+\frac{1}{2}$, there exists a constant $C$ such that

$$
\|v\|_{\sigma} \leqslant C h_{\Delta}^{\tau-\sigma}\|v\|_{\tau}, \quad \text { for } v \in S_{d}(\Delta)
$$

for all $\rho$-quasiuniform meshes $\Delta$. We will comment briefly on how (2.1.30) is established assuming henceforth that all meshes appearing are $\rho$-quasiuniform for some fixed $\rho$.

First, (2.1.30) is easily established in the case $\sigma=\tau+1=d$, using the equivalence of norms on the space of polynomials of degree $d$ and a scaling argument [14], [74]. Now, letting $P_{r}$ denote the orthogonal projection of $H^{r}$ onto $S_{d}(\Delta)$, we have from this case that

$$
\left\|P_{d-1} v\right\|_{d} \leqslant C h_{\Delta}^{-1}\|v\|_{d-1}, \quad v \in H^{d} .
$$

Again using this inverse inequality, comparing $P_{d-1} v$ to $P_{d} v$, and using the easily established error estimate $\left\|P_{d} v-v\right\|_{d-1} \leqslant C h\|v\|_{d}$, we also get

$$
\left\|P_{d-1} v\right\|_{d} \leqslant C\|v\|_{d}, \quad \text { for } v \in H^{d} .
$$

Using duality with respect to the $H^{d-1}$ inner product (2.1.2) it follows that

$$
\left\|P_{d-1} v\right\|_{d-1} \leqslant C h_{\Delta}^{-1}\|v\|_{d-2}
$$

and

$$
\left\|P_{d-1} v\right\|_{d-2} \leqslant C\|v\|_{d-2},
$$

for $v \in H^{d-2}$. Now, for $\tau \in[d-1, d]$,

$$
\begin{aligned}
\left\|P_{d-1} v\right\|_{d}= & \sup _{w \neq 0} \frac{\left\langle P_{d-1} v, w\right\rangle_{d-1}}{\|w\|_{d-2}} \\
= & \sup _{w \neq 0} \frac{\left\langle v, P_{d-1} w\right\rangle_{d-1}}{\left\|P_{d-1} w\right\|_{d-1}^{d-\tau}\left\|P_{d-1} w\right\|_{d-2}^{\tau-d+1}} \\
& \cdot\left(\frac{\left\|P_{d-1} w\right\|_{d-1}}{\|w\|_{d-2}}\right)^{d-\tau}\left(\frac{\left\|P_{d-1} w\right\|_{d-2}}{\|w\|_{d-2}}\right)^{\tau-d+1} .
\end{aligned}
$$

Now the interpolation inequality

$$
\|y\|_{\beta} \leqslant\|y\|_{\gamma}^{(\delta-\beta) /(\delta-\gamma)}\|y\|_{\delta}^{(\beta-\gamma) /(\delta-\gamma)}, \quad y \in H^{\delta},
$$

holds for any real numbers $\gamma \leqslant \beta \leqslant \delta$ as follows directly from (2.1.1). Applying this to (2.1.33) with $\delta=d-1, \gamma=d-2, \beta=2 d-\tau-2$ and using (2.1.31) and (2.1.32), we deduce that

$$
\left\|P_{d-1} v\right\|_{d} \leqslant C h_{\Delta}^{\tau-d} \sup _{y \neq 0} \frac{\langle v, y\rangle_{d-1}}{\|y\|_{2 d-\tau-2}}=C h_{\Delta}^{\tau-d}\|v\|_{\tau}, \quad v \in H^{d-2},
$$


which gives (2.1.30) in the case $\sigma=d, \tau \in[d-1, d]$. If $\tau \leqslant d-1$, then, by (2.1.34) and the already completed case $\sigma=\tau+1=d$,

$$
\|v\|_{d-1}^{d-\tau} \leqslant\|v\|_{d}^{d-\tau-1}\|v\|_{\tau} \leqslant C h_{\Delta}^{\tau-d+1}\|v\|_{d-1}^{d-\tau-1}\|v\|_{\tau}, \quad v \in S_{d}(\Delta)
$$

so

$$
\|v\|_{d} \leqslant C h_{\Delta}^{-1}\|v\|_{d-1} \leqslant C h_{\Delta}^{\tau-d}\|v\|_{\tau}, \quad v \in S_{d}(\Delta) .
$$

This establishes the inverse property (2.1.30) for $\tau \leqslant \sigma=d$. Now if $\tau \leqslant \sigma \leqslant d$, we then have, by (2.1.34),

$$
\|v\|_{\sigma} \leqslant\|v\|_{d}^{(\sigma-\tau) /(d-\tau)}\|v\|_{\tau}^{(d-\sigma) /(d-\tau)} \leqslant C h_{\Delta}^{\tau-\sigma}\|v\|_{\tau}, \quad v \in S_{d}(\Delta),
$$

and so the proof of (2.1.30) is complete except for $\sigma \in\left(d, d+\frac{1}{2}\right)$. This case, like the corresponding case for the approximation properties, does not appear to be in the literature (see the discussion following (2.1.4)). It is easily established for uniform meshes, but a more involved computation appears necessary for general quasiuniform meshes (see also [106]). We also note that we do not require this case if the order $2 \alpha$ is an integer, as it is in most applications.

THEOREM 2.1.7. Let $s$ and $t$ be real numbers with $j+\alpha \leqslant t \leqslant s \leqslant 2 j, t<2 j-\frac{1}{2}$ and suppose that the solution $u \in H^{s}$. Assume that the inverse property (2.1.30) holds for all meshes under consideration with $\tau=j+\alpha, \sigma=t$. Then

$$
\left\|u-u_{\Delta}\right\|_{t} \leqslant C h_{\Delta}^{s-t}\|u\|_{s} .
$$

Proof. Let $P_{\Delta}: H^{s} \rightarrow S_{d}(\Delta)$ be an operator providing optimal order approximation in $H^{t}$ and $H^{j+\alpha}$ [23], [46]:

$$
h_{\Delta}^{t}\left\|u-P_{\Delta} u\right\|_{t}+h_{\Delta}^{j+\alpha} H u-P_{\Delta} u\left\|_{j+\alpha} \leqslant C h_{\Delta}^{s}\right\| u \|_{s} .
$$

Then

$$
\begin{aligned}
\left\|u-u_{\Delta}\right\|_{t} & \leqslant\left\|u-P_{\Delta} u\right\|_{t}+\left\|u_{\Delta}-P_{\Delta} u\right\|_{t} \\
& \leqslant\left\|u-P_{\Delta} u\right\|_{t}+C h_{\Delta}^{j+\alpha-t}\left(\left\|u-u_{\Delta}\right\|_{j+\alpha}+\left\|u-P_{\Delta} u\right\|_{j+\alpha}\right) \\
& \leqslant C h_{\Delta}^{s-t}\|u\|_{s} . \quad \square
\end{aligned}
$$

Having completed the asymptotic analysis of the collocation method, we now compare it to a standard Galerkin procedure. We have shown that the collocation procedure is essentially equivalent to a Galerkin procedure based on the $H^{j}$ inner product. It followed that this process is stable in $H^{j+\alpha}$ and so the collocation solution provides quasioptimal approximation in that space (Theorem 2.1.5). Moreover we have derived optimal estimates (i.e., estimates of optimal order with minimal smoothness assumptions on the solution) in $H^{t}$ for $t \in[2 \alpha, j+\alpha]$ (Theorem 2.1.6), and, assuming quasiuniformity, for $t \in\left[j+\alpha, 2 j-\frac{1}{2}\right.$ ) (Theorem 2.1.7).

The standard Galerkin method defines $\bar{u}_{\Delta} \in S_{d}(\Delta)$ by the equations

$$
\left\langle A \bar{u}_{\Delta}, v\right\rangle_{0}=\langle f, v\rangle_{0} \text { for all } v \in S_{d}(\Delta) \text {. }
$$

The usual analysis is based on 0-coercivity of the operator $A$ (see (2.1.6)). Under this assumption the method is easily shown to be stable in $H^{\alpha}$, whence $\bar{u}_{\Delta}$ provides quasioptimal approximation in that space [15], [92]. Using duality, optimal estimates can also be derived in $H^{t}$ for $t \in[2 \alpha-2 j, \alpha]$, and, assuming quasiuniformity, for $t \in\left[\alpha, 2 j-\frac{1}{2}\right.$ ) as well [15], [54]. Note that if the mesh is quasiuniform the range of 
spaces in which optimal estimates hold for the Galerkin method strictly contains the corresponding range for the collocation method. Assuming a smooth solution, the highest rate of convergence achieved by the collocation method is $O\left(h^{2 j-2 \alpha}\right)$ in $H^{2 \alpha}$, while the Galerkin solution converges with rate $O\left(h^{4 j-2 \alpha}\right)$ in $H^{2 \alpha-2 j}$. This situation is summarized in Figure 1.

In boundary integral methods for problems in potential theory and continuum mechanics, the curve $\Gamma$ is the boundary of a two-dimensional domain, and the physical fields are defined by integrals of $u$ over $\Gamma$. For points not on $\Gamma$ the kernel will generally be smooth, and so the approximate value of the field will converge at a rate equal to the highest rate of convergence achieved by the approximate solution to $u$. Moreover numerical experiments also show superconvergence at the same rates at nodal points [48], [51], [64], [65]. Hence, to obtain the same order of superconvergence as the standard Galerkin method, the collocation method requires splines whose degree exceed by one twice the degree of the splines employed for the Galerkin method. On the other hand the construction of the stiffness matrix for the Galerkin method for integral equations requires the evaluation of double integrals while the collocation method ony requires single integrals.

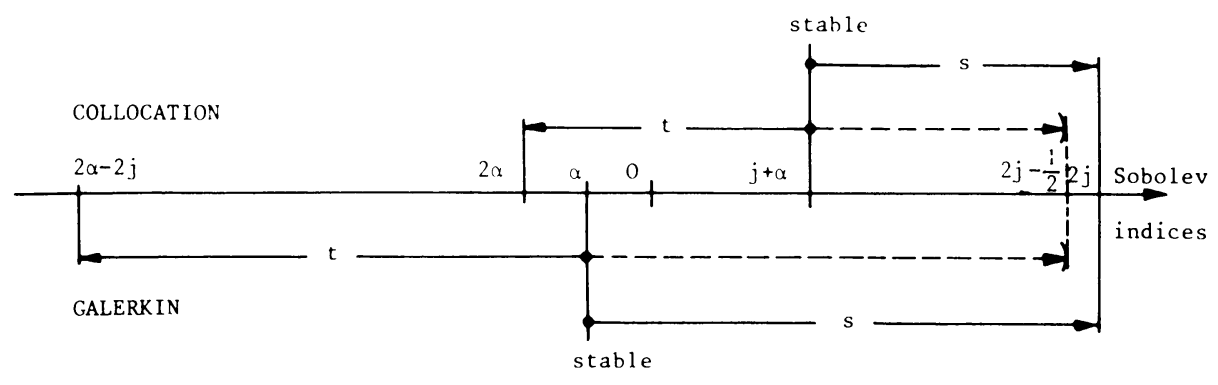

The case $\alpha=0 \quad(j+\alpha \backslash 0$ pictured, $j+\alpha \cdot 0$ also possible $)$

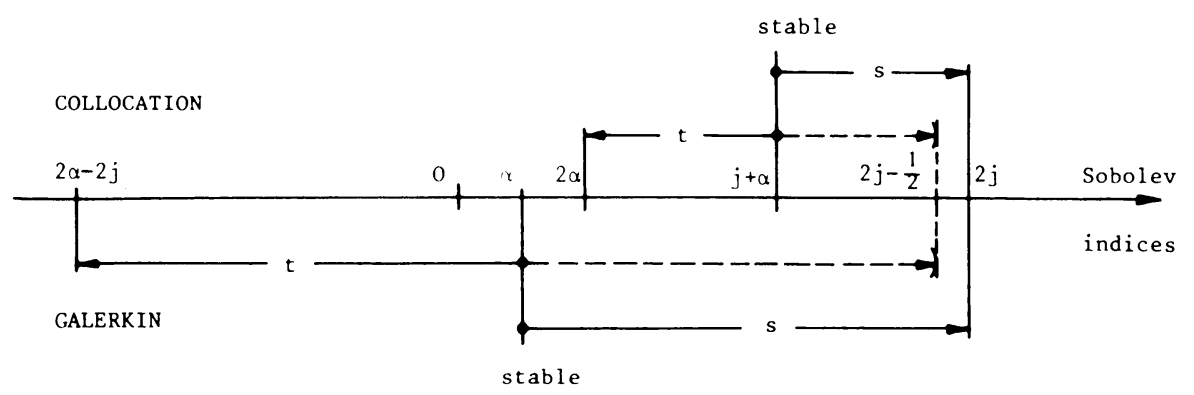

The case $\alpha \geq 0$

FIGURE 1

The indices $t \leqslant s$ for which $\left\|u-u_{\Delta}\right\|_{t} \leqslant C h_{\Delta}^{s-t}\|u\|_{s}$.

Dashed lines indicate estimates requiring a quasiuniform mesh family.

We conclude this section with a determination of the condition number of the matrix arising in the collocation method. Let $q_{d}$ denote the interpolation operator onto $S_{d}(\Delta)$. Then the collocation equations (2.1.5) may be written as

$$
g_{d} A u_{\Delta}=g_{d} f
$$


and the linear operator which must be inverted to determine $u_{\Delta}$ is $\mathscr{G}_{d} A: S_{d}(\Delta) \rightarrow$ $S_{d}(\Delta)$. In the following theorem we show that for quasiuniform meshes the $H^{0}$-condition number of this operator is bounded by $O\left(h_{\Delta}^{-2|\alpha|}\right)$.

THEOREM 2.1.8. Assume that $A$ is an isomorphism of $H^{r+\alpha}$ onto $H^{r-\alpha}$ for all real $r$, and assume that the inverse properties (2.1.30) hold for all meshes under consideration. Then there exists a constant $C$ such that for any splines $v, w \in S_{d}(\Delta)$ satisfying $\oint_{d} A v=w$, there holds

$$
\|v\|_{0} \leqslant C h_{\Delta}^{2 \alpha}\|w\|_{0}, \quad\|w\|_{0} \leqslant C\|v\|_{0} \quad \text { if } \alpha \leqslant 0
$$

and

$$
\|v\|_{0} \leqslant C\|w\|_{0}, \quad\|w\|_{0} \leqslant C h_{\Delta}^{-2 \alpha}\|v\|_{0} \quad \text { if } \alpha>0
$$

Thus the $H^{0}$-condition number of $\oint_{d} A: S_{d}(\Delta) \rightarrow S_{d}(\Delta)$ is bounded by $C h_{\Delta}^{-2|\alpha|}$.

Proof. The equation (2.1.36) is equivalent to

$$
g_{d} A v\left(x_{i}\right)=w\left(x_{i}\right)=9_{d} w\left(x_{i}\right), \quad i=1, \ldots, n,
$$

where we choose $\tilde{d}$ satisfying $\tilde{d}>\max \left(-2 \alpha-\frac{1}{2}, j-\alpha-\frac{1}{2}\right)$ and $\tilde{d} \geqslant d$.

(i) Suppose $\alpha \leqslant 0$. Then we may apply (2.1.22) with $t=0, s=j+\alpha$ if $j+\alpha \geqslant 0$, and Theorem 2.17 with $t=s=0$ if $j+\alpha<0$ to get

$$
\left\|v-A^{-1} \mathscr{g}_{d} w\right\|_{0} \leqslant C h_{\Delta}^{s}\left\|A^{-1} g_{d} w\right\|_{s}
$$

and, with the boundedness of $A^{-1}$,

$$
\|v\|_{0} \leqslant C_{1}\left\|{ }_{d} w\right\|_{-2 \alpha}+C_{2} h_{\Delta}^{s}\left\|{ }^{9} w\right\|_{s-2 \alpha} .
$$

For $\oint_{d} w \in S_{d}(\Delta)$ we apply inverse properties and boundedness of $\mathscr{G}_{d}$ on $S_{d}(\Delta)$,

$$
\|v\|_{0} \leqslant C h_{\Delta}^{2 \alpha}\left\|9_{d} w\right\|_{0} \leqslant C^{\prime} h_{\Delta}^{2 \alpha}\|w\|_{0} .
$$

Next we have

$$
\|w\|_{0} \leqslant\|A v\|_{0}+\left\|\left(9_{d}-I\right) A v\right\|_{0} \leqslant c_{1}\|v\|_{2 \alpha}+c_{2} h_{\Delta}^{s}\|A v\|_{s},
$$

where now $s=j-\alpha$ for $j+\alpha \geqslant 0$ and $s=1$ for $j+\alpha<0$. Then continuity of $A$ and the inverse property for $S_{d}(\Delta)$ imply

$$
\|w\|_{0} \leqslant c_{1}\|v\|_{2 \alpha}+c_{2} h_{\Delta}^{s}\|v\|_{s+2 \alpha} \leqslant c\|v\|_{2 \alpha} \leqslant c\|v\|_{0} .
$$

(ii) Now if $\alpha>0$, we apply the triangle inequality, the boundedness of $A^{-1}$, (2.1.22), and an inverse property to get

$$
\begin{aligned}
\|v\|_{0} & \leqslant\left\|A^{-1} w\right\|_{0}+\left\|v-A^{-1} w\right\|_{0} \leqslant C\|w\|_{-2 \alpha}+\left\|v-A^{-1} w\right\|_{2 \alpha} \\
& \leqslant C\left(\|w\|_{0}+h_{\Delta}^{j-\alpha}\left\|A^{-1} w\right\|_{j+\alpha}\right) \leqslant C\left(\|w\|_{0}+h_{\Delta}^{j-\alpha}\|w\|_{j-\alpha}\right) \leqslant C\|w\|_{0} .
\end{aligned}
$$

For continuity we have

$$
\begin{aligned}
\|w\|_{0} & =\left\|\Phi_{d} A v\right\|_{0} \leqslant\|A v\|_{0}+\left\|\left(g_{d}-I\right) A v\right\|_{0} \\
& \leqslant c_{1}\|v\|_{2 \alpha}+c_{2} h_{\Delta}^{j-\alpha}\|A v\|_{j-\alpha} \leqslant c_{1}\|v\|_{2 \alpha}+c_{2} h_{\Delta}^{j-\alpha}\|v\|_{j+\alpha}
\end{aligned}
$$

and, with inverse properties, $\|w\|_{0} \leqslant C h_{\Delta}^{-2 \alpha}\|v\|_{0}$.

Remark. The bounds on the condition number given above are the same as those for Galerkin's method [100]. As for the Galerkin-collocation method in [50], [100] they provide the basic estimates for the analysis for the fully discretized collocation with numerical quadrature [103] based on the Strang lemma [39]. 
2.2. Collocation of Systems of Equations of the Same Order. In this section we extend the analysis of the last section to systems of equations of the form (2.1). For $s \in \mathbf{R}$ and integers $p>0, q \geqslant 0$, set $\mathfrak{H}^{s}=\left(H^{s}\right)^{p} \times \mathbf{R}^{q}$. Let

$$
\mathbb{Q}=\left(\begin{array}{ll}
A & B \\
\Lambda & 0
\end{array}\right): \mathfrak{K}^{j+\alpha} \rightarrow \mathfrak{K}^{j-\alpha} .
$$

where $A$ is a $p \times p$ matrix of bounded linear operators $H^{j+\alpha} \rightarrow H^{j-\alpha}, B$ is a $p \times q$ matrix of functions in $H^{j-\alpha}$, and $\Lambda$ is a $q \times p$ matrix of bounded real-valued linear functionals on $H^{j+\alpha}$. We again assume the relation (A.1) between the order $2 \alpha$ of the operator and the degree $d=2 j-1$ of the collocating splines. In place of (A.2) and (A.3) we assume

$$
\mathbb{Q}: \mathfrak{H}^{j+\alpha} \rightarrow \mathfrak{H}^{j-\alpha} \text { is an isomorphism }
$$

and

Q satisfies the Gårding inequality

$$
\langle Q \mathbf{u}, \mathbf{u}\rangle_{\mathscr{H}^{\prime}} \geqslant \gamma\|\mathbf{u}\|_{\mathfrak{H}^{\prime+\alpha}}^{2}-\langle\mathcal{K} \mathbf{u}, \mathbf{u}\rangle_{\mathcal{H}^{\prime}}, \quad \mathbf{u} \in \mathcal{H}^{j+\alpha},
$$

where $\gamma>0$ and $\mathscr{K}: \mathscr{H}^{j+\alpha} \rightarrow \mathscr{H}^{j-\alpha}$ is a compact operator.

Here the spaces $\mathcal{H}^{s}$ are equipped with the natural product norms and inner products.

Remark. Since

$$
\mathcal{Q}=\left(\begin{array}{ll}
A & 0 \\
0 & 0
\end{array}\right)+\left(\begin{array}{cc}
0 & B \\
\Lambda & 0
\end{array}\right)
$$

and the second matrix on the right-hand side of this equation represents an operator of finite rank, to verify (A.3) it suffices to show that

$$
\langle A u, u\rangle_{j} \geqslant \gamma\|u\|_{j+\alpha}^{2}-\langle K u, u\rangle_{j}, \quad u \in\left(H^{j+\alpha}\right)^{p},
$$

for some $\gamma>0$ and $K:\left(H^{j+\alpha}\right)^{p} \rightarrow\left(H^{j-\alpha}\right)^{p}$ compact. (Here $\langle\cdot, \cdot\rangle_{j}$ and $\|\cdot\|_{j}$ denote the inner product and norm in $\left(H^{j}\right)^{p}$.)

Given $\mathbf{f}=(f, \beta) \in \mathcal{H}^{j-\alpha}$ we wish to approximate the solution $\mathbf{u}=(u, \omega) \in \mathcal{H}^{j+\alpha}$ of (2.1) or, equivalently, of the equation

$$
Q \mathbf{u}=\mathbf{f} .
$$

Setting $\mathcal{S}_{d}(\Delta)=\left(S_{d}(\Delta)\right)^{p} \times \mathbf{R}^{q} \subseteq \mathcal{H}^{j+\alpha}$, the collocation solution $\mathbf{u}_{\Delta}=\left(u_{\Delta}, \omega_{\Delta}\right) \in$ $\delta_{d}(\Delta)$ is defined by the equations

$$
\begin{gathered}
A u_{\Delta}\left(x_{i}\right)+B \omega_{\Delta}\left(x_{i}\right)=f\left(x_{i}\right), \quad i=1, \ldots, n, \\
\Lambda u_{\Delta}=\beta .
\end{gathered}
$$

Now let $\mathscr{G}_{\Delta}^{ \pm}: \mathcal{K}^{j-\alpha} \rightarrow \mathcal{H}^{j-\alpha}$ be defined by $\mathscr{q}_{\Delta}^{ \pm}(u, \omega)=\left(u \pm J_{\Delta} u \mp J u, \omega\right)$, the operators $J$ and $J_{\Delta}$ being applied componentwise. Set $\mathbb{Q}_{\Delta}=\mathscr{G}_{\Delta}^{+} Q$. The following analogue of Theorem 2.1.2 follows easily from Theorem 2.1.1.

THEOREM 2.2.1. A function $\mathbf{u}_{\Delta} \in \mathcal{S}_{d}(\Delta)$ satisfies the collocation equations (2.2.1) if and only if it satisfies the Galerkin equations

$$
\left\langle Q_{\Delta} \mathbf{u}_{\Delta}, \mathbf{v}\right\rangle_{\mathscr{K}^{j}}=\left\langle Q_{\Delta} \mathbf{u}, \mathbf{v}\right\rangle_{\mathcal{C}^{j}}, \quad \mathbf{v} \in \delta_{d}(\Delta) .
$$

Lemma 2.2.2 and Theorems 2.2.3 and 2.2.4 which assert the uniform boundedness, stability, and quasioptimality of the collocation procedure may now be proved in a 
manner entirely analogous to the case of a single equation, and so their proofs are omitted.

LEMMA 2.2.2. The operator $Q_{\Delta}$ is invertible with inverse $Q_{\Delta}^{-1}=Q^{-1} G_{\Delta}^{-}$. Moreover there exists a constant $C$ such that

$$
\left\|\mathbb{Q}_{\Delta}\right\|_{\mathscr{K}^{\jmath+\alpha}, \mathfrak{K}^{\prime-\alpha}}+\left\|\mathbb{Q}_{\Delta}^{-1}\right\|_{\mathscr{K}^{j^{\prime-\alpha}, \mathcal{K}^{\prime+\alpha}}} \leqslant C
$$

for all partitions $\Delta$.

THEOREM 2.2.3. There exist positive constants $C$ and $h_{0}$ such that if $\Delta$ is a partition for which $h_{\Delta} \leqslant h_{0}$, then

$$
\inf _{\substack{\mathbf{v} \in \delta_{d}(\Delta) \\\|\mathbf{v}\|_{K^{\prime+\alpha}}=1}} \sup _{\substack{\mathbf{z} \in \mathcal{S}_{d}(\Delta) \\\|\mathbf{z}\|_{\aleph^{\prime}} j^{+\alpha}=1}}\left\langle Q_{\Delta} \mathbf{v}, \mathbf{z}\right\rangle_{\mathcal{K}} \geqslant C .
$$

THEOREM 2.2.4. There exist positive constants $C$ and $h_{0}$ such that for any partition $\Delta$ with $h_{\Delta} \leqslant h_{0}$ there is a unique solution $\left(u_{\Delta}, \omega_{\Delta}\right) \in \mathcal{S}_{d}(\Delta)$ of the collocation equation (2.2.1). Moreover

$$
\left\|u-u_{\Delta}\right\|_{j+\alpha}+\left|\omega-\omega_{\Delta}\right| \leqslant C \inf _{v \in\left(S_{d}(\Delta)\right)^{p}}\|u-v\|_{j+\alpha} .
$$

If in addition $u \in\left(H^{s}\right)^{p}$ for some $s \in[j+\alpha, 2 j]$, then

$$
\left\|u-u_{\Delta}\right\|_{j+\alpha}+\left|\omega-\omega_{\Delta}\right| \leqslant C h_{\Delta}^{s-j-\alpha}\|u\|_{s} .
$$

To state the analogue of Theorem 2.1.6 we introduce the $j$-adjoint of an operator $\mathscr{B}: \mathcal{H}^{s} \rightarrow \mathcal{H}^{t}$ as the operator $\mathscr{B}^{*}: \mathcal{H}^{2 j-t} \rightarrow \mathcal{H}^{2 j-s}$ defined by

$$
\left\langle\mathbf{v}, \mathscr{B}^{*} \mathbf{w}\right\rangle_{\mathcal{K}^{\prime}}=\langle\mathscr{B} \mathbf{v}, \mathbf{w}\rangle_{\mathscr{K}^{\prime}}, \quad \mathbf{v} \in \mathcal{H}^{s}, \mathbf{w} \in \mathcal{H}^{2 j-t} .
$$

Also, for $s>j+\alpha$ we say that an isomorphism $\mathscr{B}: \mathscr{H}^{j+\alpha} \rightarrow \mathcal{H}^{j-\alpha}$ is $s$-regular if $\mathscr{B}^{-1}$ maps $\mathcal{H}^{s-2 \alpha}$ boundedly into $\mathcal{H}^{s}$. The proof of the following theorem is completely analogous to that of Theorem 2.1.6.

THEOREM 2.2.5. Let $t \in[2 \alpha, j+\alpha]$ and assume that $\mathbb{Q}^{*}$ is $(2(j+\alpha)-t)$-regular. In case $t \leqslant 2 \alpha+\frac{1}{2}$ and $j>\alpha+2$, assume in addition that $Q$ maps $\mathcal{H}^{2+2 \alpha}$ boundedly into $\mathcal{H}^{2}$. Then

$$
\left\|u-u_{\Delta}\right\|_{t}+\left|\omega-\omega_{\Delta}\right| \leqslant C h_{\Delta}^{j+\alpha-t}\left\|u-u_{\Delta}\right\|_{j+\alpha} .
$$

If in addition $u \in\left(H^{s}\right)^{p}$ for some $s \in[j+\alpha, 2 j]$ then

$$
\left\|u-u_{\Delta}\right\|_{t}+\left|\omega-\omega_{\Delta}\right| \leqslant C h^{s-t}\|u\|_{s} .
$$

Finally, the inverse inequality (2.1.30) clearly implies the analogous result for $\mathbf{v} \in \mathcal{S}_{d}(\Delta)$, namely that for $\tau \leqslant \sigma<d+\frac{1}{2}$,

$$
\|\mathbf{v}\|_{\sigma} \leqslant C h_{\Delta}^{\tau-\sigma}\|\mathbf{v}\|_{\tau}, \quad \mathbf{v} \in \delta_{d}(\Delta) .
$$

It is therefore straightforward to derive the following analogue of Theorem 2.1.7.

THEOREM 2.2.6. Let $s$ and $t$ be real numbers with $j+\alpha \leqslant t \leqslant s \leqslant 2 j, t<2 j-\frac{1}{2}$ and suppose that the solution $\mathbf{u} \in \mathcal{H}^{s}$. Assume that the inverse property (2.2.3) holds for all meshes under consideration with $\tau=j+\alpha, \sigma=s$. Then

$$
\left\|u-u_{\Delta}\right\|_{t} \leqslant C h_{\Delta}^{s-t}\|u\|_{s} .
$$


We conclude this section by noting that, as in the case of a single equation the highest rate of convergence of the collocation method in any Sobolev space is $O\left(h^{2 j-2 \alpha}\right)$. The usual analysis for standard Galerkin methods [54], [92] can be simply adapted to systems of the form (2.1) (under a suitable coercivity hypothesis) to produce optimal error estimates (2.2.2), with $t \in[2 \alpha-2 j, \alpha]$ and $s \in[\alpha, 2 j]$ for a standard Galerkin procedure. These include estimates in Sobolev spaces of lower index and therefore of higher order than for the collocation method. The situation is altogether analogous to that summarized in Figure 1 for the scalar case. Note that

$$
\left|\omega-\omega_{\Delta}\right| \leqslant C h_{\Delta}^{s-t}\|u\|_{s}
$$

with $t=2 \alpha, s \in[j+\alpha, 2 j]$ for collocation and $t=2 \alpha-2 j, s \in[\alpha, 2 j]$ for standard Galerkin.

2.3. Applications and Examples. Since all the problems from applications considered here may be formulated in the framework of pseudodifferential operators, we begin by determining a class of such operators which satisfy assumptions (A.2, 3) or $(Q .2,3)$ of Sections 2.1 and 2.2 , respectively. To this end let $A$ be a $p \times p$ matrix of real pseudodifferential operators on the closed curve $\Gamma$ of order $2 \alpha, \alpha \in \mathbf{R}$. So $A$ maps real-valued functions to real-valued functions. (See [97], [90] for the general theory of pseudodifferential operators.) For simplicity we assume that $\Gamma$ is a $C^{\infty}$-curve, although for specific results this can be weakened significantly. Then $A$ has a principal symbol $a_{0}(z, \xi)$ subject to any fixed local parametrization $z=z(t)$ of $\Gamma$ [97, p. 54 ff.]. Here $a_{0}$ : $\operatorname{image}(z) \times \mathbf{R} \rightarrow \mathbf{C}^{p \times p}$ is homogeneous of degree $2 \alpha$ with respect to $\xi$ for $|\xi| \geqslant 1$. The significance of the principal symbol is this: if $u$ is a function on $\Gamma$ supported in the image of a single coordinate chart, then

$$
A u(z(s))=\frac{1}{2 \pi} \int_{\mathbf{R}} a_{0}(z(s), \xi) e^{i s \xi} \int_{\mathbf{R}} e^{-i t \xi} u(z(t)) d t d \xi+K u(z(s)),
$$

where $K$ maps $\left(H^{r+\alpha}\right)^{p}$ boundedly into $\left(H^{r-\alpha+\varepsilon}\right)^{p}$ for some fixed positive $\varepsilon$ and every $r \in \mathbf{R}$, i.e., $K$ is an operator of order less than $2 \alpha$.

In order to assure the Gårding inequality in $(@ .3)$ we will assume that $A$ is strongly elliptic [92], [100], i.e., that there exists a smooth function $\theta: \Gamma \rightarrow \mathbf{C}^{p \times p}$ and $\gamma>0$ such that, for each chart $z(t)$ in some covering of $\Gamma$ and corresponding principal symbol $a_{0}$, we have

$$
\operatorname{Re} \zeta^{T} \theta(y) a_{0}(y, \xi) \bar{\zeta} \geqslant \gamma|\zeta|^{2}, \quad \zeta \in \mathbf{C}^{p}, y \in \operatorname{image}(z),|\xi|=1 .
$$

Note that since $A$ is a real operator, we have $a_{0}(y,-\xi)=\overline{a_{0}(y, \xi)}$. Consequently (2.3.1) remains valid with $\theta$ replaced by its real part. Hence, without loss of generality, we assume that $\theta$ is in fact real-valued.

THEOREM 2.3.1. Let A be a strongly elliptic matrix of pseudodifferential operators on $\Gamma$ and set

$$
\mathbf{A}=A \theta
$$

where $\theta$ is as in (2.3.1). Then for any $r \in \mathbf{R}, \mathbf{A}$ maps $\left(H^{r+\alpha}\right)^{p}$ boundedly into $\left(H^{r-\alpha}\right)^{p}$ and is a Fredholm mapping of index zero. Moreover, for any nonnegative integer $j$ there exist real constants $\gamma>0$ and $C$ such that

$$
\langle\mathbf{A} v, v\rangle_{j} \geqslant \gamma\|v\|_{j+\alpha}^{2}-C\|v\|_{j+\alpha-\varepsilon}^{2}, \quad v \in\left(H^{j+\alpha}\right)^{p}
$$


Proof. From homogeneity of the principal symbol it follows that $\mathbf{A}$ maps $\left(H^{r+\alpha}\right)^{p}$ boundedly into $\left(H^{r-\alpha}\right)^{p}$. Now let $j$ be a positive integer and define

$$
D u(z(t))=(-1)^{j} \frac{d^{2 j}}{d t^{2 j}} \mathbf{A} u(z(t))
$$

Then $D$ is a matrix of pseudodifferential operators of order $2(j+\alpha)$ with principal symbol

$$
d_{0}(z, \xi)=\xi^{2 j} \theta(z) a_{0}(z, \xi),
$$

and so is strongly elliptic. Integrating by parts in the $H^{j}$ inner product and applying the results of [58] to $D$, we have for $v \in\left(H^{j+\alpha}\right)^{p}$

$$
\begin{aligned}
\langle\mathbf{A} v, v\rangle_{j} & =\langle D v, v\rangle_{0}+J(\mathbf{A} v) \cdot J v \\
& \geqslant \gamma\|v\|_{j+\alpha}^{2}-C\|v\|_{j+\alpha-\varepsilon}^{2}-|J \mathbf{A} v \| J v| \\
& \geqslant \gamma\|v\|_{j+\alpha}^{2}-C\|v\|_{j+\alpha-\varepsilon}^{2}-C\|\mathbf{A} v\|_{j-\alpha-\varepsilon}\|v\|_{j+\alpha-\varepsilon} \\
& \geqslant \gamma\|v\|_{j+\alpha}^{2}-C\|v\|_{j+\alpha-\varepsilon}^{2},
\end{aligned}
$$

for some $\gamma \geqslant 0$. That $\mathbf{A}$ is a Fredholm map of index zero $\left(H^{j+\alpha}\right)^{p} \rightarrow\left(H^{j-\alpha}\right)^{p}$ follows from this Gårding inequality [93]. This still holds true if $j$ is replaced by any real number $r$, since the Fredholm property and the index of an elliptic pseudodifferential operator is independent of $r$ [97, Chapter II, Theorem 2.5], [77], [90].

Now consider the system (2.1) with $A$ a strongly elliptic matrix of real pseudodifferential operators of order $2 \alpha$ and $B$ and $\Lambda$ as in the first paragraph of Section 2.2. Assume that for the data $f=0, \beta=0$ the only solution is the trivial one. Set

$$
\mathbb{Q}=\left(\begin{array}{cc}
\theta A & \theta B \\
\Lambda & 0
\end{array}\right)
$$

Clearly $Q$ is an invertible (since $Q$ is invertible and $\theta$ is nonsingular by (2.3.1)), i.e. (Q.2) holds with $\mathbb{Q}$ replaced by $\mathbb{Q}$. Moreover condition ( $Q$.3) with $\mathbb{Q}$ replaced by $\mathbb{Q}$ follows from Theorem 2.3.1. In view of Theorem 2.2.5 we now also establish the regularity of $\mathbb{Q}^{*}$, the $j$-adjoint of $\mathbb{Q}$.

LEMMA 2.2.3. Let $Q$ be as above and assume in addition that the components of $\Lambda$ extend continuously to $H^{2 \alpha}$. Then $\mathbb{Q}^{*}$ is $(2(j+\alpha)-t)$-regular for any $t \in[2 \alpha, j+\alpha]$.

Proof. From the definition of $\mathbb{Q}^{*}$ and $(2(j+\alpha)-t)$-regularity it clearly suffices to show that $\mathcal{Q}$ maps $\mathcal{H}^{t}$ isomorphically onto $\mathcal{H}^{t-2 \alpha}$. The hypotheses imply that $\mathcal{Q}$ is a continuous Fredholm operator of index 0, and, since it is invertible, the lemma follows.

We have now established the hypotheses of Theorems 2.2.4-2.2.6 for the operator $\mathbb{Q}$, and thus the collocation method is stable in $\mathcal{H}^{j+\alpha}$, and the full range of optimal error estimates hold, as discussed in the previous sections, for the transformed system

$$
\theta(A u+B \omega)=\theta f, \quad \Lambda u=\beta
$$


Since $\theta$ is invertible for all $z$, the transformed system is equivalent to the original one. Moreover, for the same reason, the collocation solution $\mathbf{u}_{\Delta} \in S_{d}(\Delta)$ may be determined directly from the equations

$$
\begin{gathered}
A u_{\Delta}\left(x_{i}\right)+B \omega_{\Delta}\left(x_{i}\right)=f\left(x_{i}\right), \quad i=1, \ldots, n, \\
\Lambda u_{\Delta}=\beta .
\end{gathered}
$$

Thus we have established the convergence estimates for collocation to the given system (2.1).

Remark. The boundedness and coercivity hypotheses needed to apply the standard theory of Galerkin methods follow from the assumptions above and Theorem 2.3.1. Thus the standard Galerkin method for the transformed system (2.3.2) is stable in $\mathcal{H}^{\alpha}$, and optimal order error estimates hold as indicated in Figure 1. Note however, for the Galerkin method-unlike for the collocation method-it is necessary to determine the operator $\theta$ to implement the procedure. This may be rather involved (see Section 2.3.1 and the appendix).

Remark. If the operator $A$ in (2.1) is a matrix of complex pseudodifferential operators, $\Lambda$ a matrix of complex functionals and the matrix of functions $B$ complex valued, then this system is equivalent to the larger real system

$$
\left(\begin{array}{cccc}
A_{1} & -A_{2} & B_{1} & -B_{2} \\
A_{2} & A_{1} & B_{2} & B_{1} \\
\Lambda_{1} & -\Lambda_{2} & 0 & 0 \\
\Lambda_{2} & \Lambda_{1} & &
\end{array}\right)\left(\begin{array}{l}
u_{1} \\
u_{2} \\
\omega_{1} \\
\omega_{2}
\end{array}\right)=\left(\begin{array}{l}
f_{1} \\
f_{2} \\
\beta_{1} \\
\beta_{2}
\end{array}\right)
$$

where $u_{j}, \omega_{j}, f_{j}, \beta_{j}$ represent real and imaginary parts for $j=1$ and 2 , respectively, and for $A, B$ and $\Lambda, A_{j}, B_{j}$ and $\Lambda_{j}$ represent the real operators gotten by restricting to real functions and taking real and imaginary parts. The principal symbols of $A_{1}$ and $A_{2}$ are given by

$$
\frac{1}{2}\left\{a_{0}(y, \xi)+\overline{a_{0}(y,-\xi)}\right\} \quad \text { and } \quad \frac{i}{2}\left\{a_{0}(y, \xi)-\overline{a_{0}(y,-\xi)}\right\}
$$

It follows easily that if the strong ellipticity condition (2.3.1) holds for the complex matrix of operators $A$ with principal symbol $a_{0}$ and some complex matrix $\theta=\theta_{1}+$ $i \theta_{2}$, then the real matrix of operators

$$
\left(\begin{array}{cc}
A_{1} & -A_{2} \\
A_{2} & A_{1}
\end{array}\right)
$$

is strongly elliptic with the matrix

$$
\left(\begin{array}{cc}
\theta_{1} & -\theta_{2} \\
\theta_{2} & \theta_{1}
\end{array}\right)
$$

in place of $\theta$ in (2.3.1).

It is easily verified that collocation of the complex system (2.3.3) by using complex-valued splines is equivalent to collocation of the real system (2.3.4) using real-valued splines. Having verified the strong ellipticity, we see that our convergence theory applies fully to collocation of the complex system. 
2.3.1. Integro-Differential Equations With Cauchy Kernel. Many equations arising in applications involve compositions of differential operators and singular integral operators with Cauchy kernel. These systems are of the form (2.1) with

$$
\begin{aligned}
& A_{0}(s) \frac{d^{m} u}{d s^{m}}+\sum_{j=0}^{m-1} A_{m-j}(s) \frac{d^{j} u}{d s^{j}} \\
& +\frac{1}{\pi i} \int_{\Gamma}\left\{C_{0}(s, \sigma) \frac{d^{m} u}{d \sigma^{m}}(\sigma)+\sum_{j=0}^{m-1} C_{m-j}(s, \sigma) \frac{d^{j} u}{d \sigma^{j}}(\sigma)\right\} \frac{d \zeta}{\zeta-z(s)} \\
& +\int_{\Gamma} L(s, \sigma) u(\sigma) d \sigma+\sum_{k=1}^{q} \omega_{k} B_{k}(s)=f(s), \\
& \Lambda u=\int_{\Gamma} \lambda_{0}(s) u(s) d s=\beta, \quad m \in \mathbf{N}_{0},
\end{aligned}
$$

where $\zeta=z_{1}(\sigma)+i z_{2}(\sigma)$ and where $s$, respectively, $\sigma$ denotes the arclength on $\Gamma$. Here $A_{l}, C_{l}, L$, and $\lambda_{0}$ are sufficiently smooth complex matrix-valued functions. (See [3], [69, VII, Section 1], [71, Section 117].) The principal symbol of this operator subject to a local parametrization by arc length $s$ is given by the matrix-valued function

$$
a_{0}(s, \xi)=i^{m} \xi^{m}\left(A_{0}(s)+C_{0}(s, s) \frac{\xi}{|\xi|}\right)
$$

with order $2 \alpha=m$. Hence, in order that assumption (A.1) for the collocation be satisfied, we must have $d=2 j-1>m$. We consider the cases of even and odd order operators separately.

The case of even $m$. In the case $m$ is even, $\alpha$ is a nonnegative integer and the strong ellipticity condition (2.3.1) now reads as

$$
(-1)^{\alpha} \operatorname{Re} \zeta^{T} \theta(s)\left(A_{0}(s) \pm C_{0}(s, s)\right) \bar{\zeta} \geqslant \gamma|\zeta|^{2},
$$

which must hold for both signs. In [80, Lemma 4.4] and [81, Lemma 2.9], Prössdorf and Schmidt proved that the strong ellipticity condition (2.3.5) holds if and only if

$$
\operatorname{det}\left(A_{0}(s)+\lambda C_{0}(s, s)\right) \neq 0 \text { for all } \lambda \in[-1,1] \text {. }
$$

Note that (2.3.6) precludes the possibility that $A_{0} \equiv 0$.

Remark. In [81] Prössdorf and Schmidt also construct the matrix $\theta(s)$ occurring in (2.3.5) assuming that (2.3.6) holds. Their construction (which is summarized in the appendix below) is rather involved. Hence, if $\theta$ is not given explicitly with the problem, the Galerkin method for (2.3.2) may be impractical, whereas the collocation method (2.3.3) does not require knowledge of $\theta$.

In the special case $C \equiv 0$, the operator (2.3.4) reduces to a system of ordinary differential operators of even order $2 \alpha$. The ellipticity condition is definiteness of the leading coefficient matrix $A_{0}$, and our results provide new error estimates for the nodal collocation of ordinary differential equations with periodic side conditions by odd degree splines.

Singular integral equations. In the case $\alpha=m=0$, (2.3.4) reduces to a system of singular integral equations with Cauchy kernel. For collocation by piecewise linear functions, i.e., $j=d=1$, we strengthen the convergence result from [80, Theorem 1] 
and [81]. In these papers an $L^{2}$-stability result is proved for strictly uniform meshes and is applied to give an $O(h|\log h|)$ convergence rate in an appropriate Hölder space. We proved instead estimates in Sobolev spaces without restrictions on the mesh, including an $O\left(h^{2}\right)$ rate in $L^{2}$. Moreover, for $j>1$, our results are completely new. On the other hand, Prössdorf and Schmidt show in [80], [81] that the strong ellipticity condition (2.3.5), (2.3.6) is also necessary for the convergence of piecewise linear collocation for singular integral equations.

Singular integral equations of this type have applications, e.g., to plane elasticity. For example for the second fundamental problem in which the stress is given on the boundary, the system of equations for the desired boundary displacement $u=$ $\left(u_{1}, u_{2}\right)$ may be written in the form $(2.1)$ with

$$
\begin{gathered}
f \text { given, } \quad \beta=0, \\
B(z)=\left(\begin{array}{ccc}
1 & 0 & z_{2} \\
0 & 1 & -z_{1}
\end{array}\right), \\
\Lambda u=\left(\int_{\Gamma} u_{1} d s, \int_{\Gamma} u_{2} d s, \int_{\Gamma} u_{1} d z_{1}+\int_{\Gamma} u_{2} d z_{2}\right), \\
A u(z)=\varepsilon u(z)-\int_{\Gamma} S(z, \zeta) u(\zeta) d \sigma, \\
S_{j k}(z, \zeta)=\frac{1}{\pi(\lambda+2 \mu)}\left(\mu \delta_{j k}+2(\lambda+\mu) \frac{\left(\zeta_{j}-z_{j}\right)\left(\zeta_{k}-z_{k}\right)}{|\zeta-z|^{2}}\right) \frac{\partial}{\partial \nu_{\zeta}} \log |z-\zeta| \\
+\frac{(-1)^{j}}{\pi}\left(1-\delta_{j k}\right) \frac{\mu}{\lambda+2 \mu} \frac{d}{d \sigma} \log |z-\zeta|, \quad j, k=1,2,
\end{gathered}
$$

where $\varepsilon= \pm 1$ corresponds to the interior, respectively, exterior problem and $B(z)$ gives rigid motions. $\mu>0$ and $\lambda>-\frac{2}{3} \mu$ are the Lamé constants, $\nu_{\zeta}$ is the exterior normal to $\Gamma$ at $\zeta=\zeta(\sigma) \in \Gamma$. The right-hand side $f$ is given from the boundary tractions (see (A.2.5)). This system is just the Somigliana identity on $\Gamma$ and can be obtained from [4], [34], [62], [69, XIV, Section 6], [70], [84] by specializing to the case of plane strain. (For plane stress replace $\lambda$ by $2 \lambda \mu /(\lambda+2 \mu)=\lambda^{*}$.) The principal symbol of the operator $A$ in (2.3.7) is

$$
a_{0}(s, \xi)=\left(\begin{array}{cc}
\varepsilon & -i \nu \frac{\xi}{|\xi|} \\
i \nu \frac{\xi}{|\xi|} & \varepsilon
\end{array}\right),
$$

where $\nu=\mu /(\lambda+2 \mu)$. It is easily seen that $0<\nu<\frac{3}{4}$ for plane strain (and $0<\nu<1$ for plane stress), hence, for $|\xi|=1, \varepsilon a_{0}(s, \xi)$ is positive definite. Therefore this system is strongly elliptic with $\theta=\varepsilon I$ in (2.3.1).

This system was used in [20, the case $\left.\Gamma_{1}=\varnothing\right]$. Similar systems can be found in [7], [8], [34], [44], [56], [60]-[63], [69, XIV, Section 6], [70], [99] and in each case the basic problems yield strongly elliptic systems of singular integral equations. Our results apply to all of these (as long as the boundary $\Gamma$ is smooth).

In [7], [8] Antes considers piecewise linear nodal collocation (our case $d=1$ ) and gives a posteriori error estimates. He does not prove convergence of the method. 
Moreover, a more detailed investigation of his estimates [7, (8.22) ff.], in light of our results, shows that they are weaker than optimal by at least one power of $h_{\Delta}$. A numerical comparison of Galerkin's method and point collocation can be found in [72], but our results do not apply since piecewise quadratic trial functions are used. Nevertheless, if our results were to hold for this case, they would be in good agreement with the experiments.

Fredholm integral equations of the second kind. A further specialization of (2.3.4) to the case $m=\alpha=0, A_{0}(x) \equiv I, C_{0} \equiv 0$, yields Fredholm integral equations of the second kind

$$
\begin{gathered}
u(s)+\int_{\Gamma} L(s, \sigma) u(\sigma) d \sigma+B(s) \omega=f(s), \\
\Lambda u=\int_{\Gamma} \lambda_{0}(\sigma) u(\sigma) d \sigma=\beta .
\end{gathered}
$$

The principal symbol is the identity matrix, so the equations are trivially strongly elliptic and our results apply. However, they can easily be obtained in this case from well-known results in [6], [28] in combination with approximation estimates (2.1.4). Further results, including superapproximation and nodal superconvergence results, can be found in [11], [12], [13], [30], [54], [78]. Such Fredholm equations arise in classical potential theory for the Laplacian and Helmholtz equation and so are applied to the computation of incompressible flows, acoustics, and classical electromagnetic scattering [83, Section 81], [56], [61]. In particular our convergence results apply to the method used in [67] to compute ideal two-dimensional flows.

The case of odd $m$.

We now turn to systems involving operators of the form (2.3.4) with odd order $m$. Then $\alpha-1 / 2$ is a nonnegative integer, and instead of (2.3.5) the strong ellipticity condition is

$$
(-1)^{\alpha-1 / 2} \operatorname{Re} i \zeta^{T} \theta(s)\left( \pm A_{0}(s)+C_{0}(s, s)\right) \bar{\zeta} \geqslant \gamma|\zeta|^{2},
$$

which again must hold for both signs.

From [81, Lemma 2.9], it follows that (2.3.8) holds if and only if

$$
\operatorname{det}\left(\lambda A_{0}(s)+C_{0}(s, s)\right) \neq 0, \quad \lambda \in[-1,1] .
$$

Hence, in the odd order case, $C_{0}$ cannot vanish.

An example is given by the normal derivatives of the double layer potential, that is the operator defined by

$$
C_{0} u(s)=\frac{1}{\pi} \frac{\partial}{\partial \nu_{z}} \int_{\Gamma} u(\sigma) \frac{\partial}{\partial \nu_{\zeta}}(\log |z-\zeta|) d \sigma,
$$

$z=z(s)$ and $\zeta=\zeta(\sigma)$ on $\Gamma$, which plays an important role in classical potential theory. Equations of the form

$$
\begin{gathered}
A_{0}(s) u(s)+C_{0} u(s)+\frac{1}{\pi i} \int_{\Gamma} C_{1}(s, \sigma) u(\sigma) \frac{d \zeta}{\zeta-z(s)} \\
+\int_{\Gamma} L(s, \sigma) u(\sigma) d \sigma+B \omega=f, \\
\Lambda u=\beta,
\end{gathered}
$$


$\zeta=\zeta(\sigma)$ on $\Gamma$, have been used more recently in numerical computations. Employing the Cauchy-Riemann equations and integration by parts, (2.3.9) can be rewritten as

$$
C_{0} u(s)=-\frac{1}{i \pi} \int_{\Gamma} \frac{d u}{d \sigma}(\sigma)\left(\frac{d z}{d s}(s) \overline{\frac{d z}{d \sigma}(\sigma)}\right) i \frac{d \zeta}{\zeta-z(s)} .
$$

The principal part of $C_{0}$ is given by

$$
u \mapsto-\frac{1}{i \pi} \int_{\Gamma} \frac{d u}{d \sigma}(\sigma) \frac{i d \zeta}{\zeta-z(s)},
$$

which is the famous Prandtl integro-differential operator of wing theory [71, Section 117], [69, VII, Section 1]. From (2.3.11) we see that (2.3.10) is a specialization of (2.3.4) to the case $A_{0}=0, C_{0}(s, s)=-i, m=1$, and the principal symbol is simply $a_{0}(s, \xi)=|\xi|$. Hence (2.3.10) is strongly elliptic, and $\theta$ in (2.3.8) can be taken to be the identity.

Applications of (2.3.10) to acoustics and electrostatics can be found in [41], [73]. In [59] an equation of the form (2.3.10) arises in elasticity problems, and piecewise linear collocation is used. Since $j=1, \alpha=\frac{1}{2}$, assumption (A.1) is violated, so our results do not apply. In [99] cubic spline collocation is applied to first order integro-differential equations of the form (2.3.4). Thus, our analysis applies as long as the boundary is smooth and mixed boundary conditions are excluded.

2.3.2. Fredholm Integral Equations of the First Kind With Logarithmic Principal Part. A large class of interior and exterior boundary value problems in two dimensions can be reduced to systems of the form

$$
\begin{gathered}
\int_{\Gamma}\left(-\frac{1}{\pi} \log |z(s)-z(\sigma)|+L(s, \sigma)\right) u(\sigma) d \sigma+B(z(s)) \omega=f(z(s)), \\
\int_{\Gamma} \lambda_{0}(\sigma) u(\sigma) d \sigma=\Lambda u=\beta,
\end{gathered}
$$

where the kernel $L$ is smoother than the logarithmic principal part. For this operator the order is $2 \alpha=-1$, and the principal symbol is $a_{0}(s, \xi)=I /|\xi|$, so the operator is strongly elliptic (and $\theta=I$ ).

Systems of this form arise in many applications and the method goes back to Fichera [37]. The scalar case with $p=q=1, B=1, \lambda_{0}=1, L=0$ is Symm's integral equation of conformal mapping [40], [45], [48], [51], [95], [100]. In [31] it is shown that the numerical conditioning is superior with $B=1, \lambda_{0}=1$ rather than $B=0, \lambda_{0}=0$ and scaling of $\Gamma$. As a system, (2.3.12) is used in viscous flow problems, particularly in connection with Stokes flows [50]-[53], electrostatics [73], [85], [86], acoustics [38], plane elasticity [20], [32], [51], [56], [73], plate bending [51], [52], and torsion problems [56], [68], [96, p. $246 \mathrm{ff}$.$] .$

In particular, for the plane first fundamental problem of elasticity with the displacements given on $\Gamma,(2.3 .12)$ specializes to

$$
p=2, \quad q=3, \quad B(z)=\left(\begin{array}{ccc}
1 & 0 & z_{2} \\
0 & 1 & -z_{1}
\end{array}\right), \quad \Lambda=\left(\begin{array}{cc}
1 & 0 \\
0 & 1 \\
z_{2} & -z_{1}
\end{array}\right)
$$

and

$$
L=\frac{\lambda+\mu}{\pi(\lambda+3 \mu)}\left(\left(\left(z_{j}-\zeta_{j}\right)\left(z_{k}-\zeta_{k}\right) /|z-\zeta|^{2}+\frac{1}{2} \delta_{j k}\right)\right)
$$


(see Appendix and [20], [34], [51], [72]). Then the unknowns in (2.3.12) are the tractions on $\Gamma . f$ is given from the displacements, see (A.2.6).

Asymptotic error analysis of Galerkin methods and their numerical implementations for (2.3.12) have appeared in many places [50], [51], [53], [54], [55], [73], [85], [86], [100]. However, only preliminary convergence results have appeared for the collocation method [1], [2], [5, p. $271 \mathrm{ff}$.$] , [98], and these do not provide quasioptimal$ estimates. Our results with $j=1$ apply in particular to numerical methods in [20], [31], [32], [68], [99].

In [48] H.-P. Hoidn reports on numerical experiments for piecewise linear and cubic collocation of Symm's integral equation. His results confirm the rates of convergence predicted by our work with great precision.

3. The Collocation of Boundary Problems in Ordinary Differential Equations. Thus far we have considered only problems with periodic side conditions. The methods of analysis employed can also be applied to the solution by collocation of two-point boundary value problems, which has generally been analyzed by quite different techniques in [9], [10], [21], [35], [66], [89]. Rather than striving for the generality of the previous chapter here we limit ourselves to the example of cubic spline collocation of the problem

$$
\begin{aligned}
A u:= & a u^{\prime \prime}+b u^{\prime}+c u=f \text { on }(0,1), \\
& u(j)=u_{j}, \quad j=0,1 .
\end{aligned}
$$

Here $a, b, c, f \in C^{1}([0,1])$ and $u_{0}, u_{1} \in \mathbf{R}$ are given. We assume that $a$ is strictly positive and that the problem (3.1) has a unique solution. The following analysis can be generalized to systems of equations, equations of higher order, splines of higher degree, and to more general boundary conditions.

We require in addition the unisolvence assumption:

$$
\text { if } v \text { is a cubic polynomial and } A^{i} v(j)=0 \text { for } i, j=0,1 \text {, then } v \equiv 0 \text {. }
$$

Let $\Delta$ denote a mesh, $0=x_{0}<x_{1}<\cdots<x_{n}=1$, of maximum subinterval length $h_{\Delta}$. Denote by $M_{3}(\Delta)$ the space of all $C^{2}$ cubic splines subordinate to $\Delta$ (but not necessarily periodic). The collocation procedure seeks $u_{\Delta} \in M_{3}(\Delta)$ satisfying

$$
\begin{aligned}
A u_{\Delta}\left(x_{j}\right) & =f\left(x_{j}\right), & j & =0,1, \ldots, n, \\
u_{\Delta}(j) & =u_{j}, & j & =0,1 .
\end{aligned}
$$

We remark that the unisolvence assumption states precisely that the collocation equations are uniquely solvable in the case of the trivial, one subinterval, mesh.

We begin by reformulating the collocation method as a nonstandard Galerkin method. Our approach is related to that in [94], but we apply the error analysis for Galerkin methods directly. Let $U$ be the unique cubic polynomial satisfying $A^{i} U(j)$ $=A^{i} u(j), i, j=0,1$. Set

$$
\tilde{H}^{3}=\left\{v \in H^{3} \mid A^{i} v(j)=0, i, j=0,1\right\}, \quad \tilde{M}_{3}(\Delta)=M_{3}(\Delta) \cap \tilde{H}^{3},
$$

where in this chapter $H^{k}$ ( $k$ integral) denotes the nonperiodic Sobolev space of index $k$ on $[0,1]$ with norm denoted by $\|\cdot\|_{k}$. Then $\tilde{u}:=u-U \in \tilde{H}^{3}$ and, supposing 
$u_{\Delta} \in M_{3}(\Delta)$ satisfies (3.2), $\tilde{u}_{\Delta}:=u_{\Delta}-U \in \tilde{M}_{3}(\Delta)$. Now, for any $\chi \in \tilde{M}_{3}(\Delta)$, $\left.\chi^{\prime \prime \prime}\right|_{\left(x_{,-1}, x_{1}\right)}$ is equal to a constant $\chi_{j} \in \mathbf{R}$, so by (3.2)

$$
\begin{aligned}
\int_{0}^{1}\left(A \tilde{u}_{\Delta}\right)^{\prime}(x) \chi^{\prime \prime \prime}(x) d x & =\left.\sum_{j=1}^{n} A \tilde{u}_{\Delta}\right|_{x_{j-1}} ^{x_{j}} \chi_{j}=\left.\sum_{j=1}^{n} A \tilde{u}\right|_{x_{j-1}} ^{x_{j}} \chi_{j} \\
& =\int_{0}^{1}(A \tilde{u})^{\prime}(x) \chi^{\prime \prime \prime}(x) d x .
\end{aligned}
$$

Defining $B: H^{3} \times H^{3} \rightarrow \mathbf{R}$ by

$$
B(v, w)=\int_{0}^{1}(A v)^{\prime}(x) w^{\prime \prime \prime}(x) d x
$$

we conclude that if $u_{\Delta} \in M_{3}(\Delta)$ satisfies the collocation equations (3.2), then $\tilde{u}_{\Delta}=u_{\Delta}-U \in \tilde{M}_{3}(\Delta)$ satisfies the Galerkin equations

$$
B\left(\tilde{u}-\tilde{u}_{\Delta}, w\right)=0, \quad w \in \tilde{M}_{3}(\Delta) .
$$

The converse also holds. Indeed, by the unisolvence assumption every piecewise constant function subordinate to $\Delta$ of mean value zero is the third derivative of some function in $\tilde{M}_{3}(\Delta)$. Hence, if $\tilde{u}_{\Delta}$ satisfies (3.3), then the node values $A\left(\tilde{u}-\tilde{u}_{\Delta}\right)\left(x_{j}\right)$ are linear in $x_{j}$ (cf. the proof of Theorem 2.1.1). Since $A\left(\tilde{u}-\tilde{u}_{\Delta}\right)(j)=0$ for $j=0,1$ the collocation equations (3.2) hold.

THEOREM 3.1. For $h_{\Delta}$ sufficiently small the collocation equations (3.2) have a unique solution $u_{\Delta} \in M_{3}(\Delta)$. Moreover there exists a constant $C$ depending only on the coefficients $a, b$, and $c$ such that

$$
\left\|u-u_{\Delta}\right\|_{3} \leqslant C \inf _{v \in M_{3}(\Delta)}\|u-v\|_{3} .
$$

Proof. Let $v \in M_{3}(\Delta)$ and let $V$ be the unique cubic polynomial satisfying $A^{i}(V+v-u)(j)=0, \quad i, j=0,1$. Then $\|V\|_{3} \leqslant C\|u-v\|_{3}$. Setting $\tilde{v}=v+V$, we have $\tilde{v} \in \tilde{M}_{3}(\Delta)$ and

$$
\|u-\tilde{v}\|_{3} \leqslant\|u-v\|_{3}+\|V\|_{3} \leqslant C\|u-v\|_{3},
$$

so it suffices to prove the quasioptimal estimate of the theorem with the infimum restricted to $v \in \tilde{M}_{3}(\Delta)$. By the standard theory for Galerkin methods [15], [16], the theorem then holds if it can be shown that

$$
\inf _{0 \neq v \in \tilde{M}_{3}(\Delta)} \sup _{0 \neq w \in \tilde{M}_{3}(\Delta)} \frac{B(v, w)}{\|v\|_{3}\|w\|_{3}} \geqslant C_{0},
$$

where $C_{0}$ is a positive constant depending only on $a, b$, and $c$. Let $v \in \tilde{M}_{3}(\Delta)$ be given. Then

$$
B(v, v) \geqslant C_{1}\|v\|_{3}^{2}-C_{2}\|v\|_{0}^{2}
$$

where $C_{1}=\frac{1}{2} \inf a>0$, and $C_{2}$ depends only on $a, b$, and $c$. Define $y$ to be the solution of the adjoint problem

$$
\begin{gathered}
A^{*} y:=(a y)^{\prime \prime}-(b y)^{\prime}+c y=v \quad \text { on }(0,1), \\
y(j)=0, \quad j=0,1 .
\end{gathered}
$$


Since the Dirichlet problem for $A$ is well-posed, so is that for $A^{*}$, so $y$ is uniquely determined and

$$
\|y\|_{0} \leqslant\|y\|_{1} \leqslant C\|v\|_{-1} \leqslant C\|v\|_{3} .
$$

Next define $z$ by the boundary value problem

$$
z^{(4)}=y \quad \text { on }(0,1), \quad A^{i} z(j)=0, \quad i, j=0,1 .
$$

By the unisolvence assumption $z$ is well-defined and

$$
\|z\|_{4} \leqslant\|y\|_{0} \leqslant C\|v\|_{3} \text {. }
$$

By construction $z \in \tilde{H}^{3}$ and

$$
B(v, z)=\|v\|_{0}^{2} .
$$

Finally let $t \in \tilde{M}^{3}(\Delta)$ be the $H^{3}$ projection of $z$ into $\tilde{M}_{3}(\Delta)$, so

$$
\|t\|_{3} \leqslant\|z\|_{3} \leqslant C\|v\|_{3},
$$

and, using (3.6),

$$
\|t-z\|_{3} \leqslant C h_{\Delta}\|z\|_{4} \leqslant C h_{\Delta}\|v\|_{3} .
$$

Setting $w=v+C_{2} t$ and combining (3.5), (3.7) and (3.9), we get

$$
B(v, w)=B(v, v)+C_{2} B(v, z)+C_{2} B(v, t-z) \geqslant C_{1}\|v\|_{3}^{2}-C h_{\Delta}\|v\|_{3}^{2} .
$$

Also, from the definition of $w$ and (3.8),

$$
\|w\|_{3} \leqslant C\|v\|_{3} \text {. }
$$

For $h_{\Delta}$ sufficiently small, (3.4) follows from (3.10) and (3.11).

COROLlaRy 3.2. If $u \in H^{4}$, then for $h_{\Delta}$ sufficiently small

$$
\left\|u-u_{\Delta}\right\|_{3} \leqslant C h_{\Delta}\|u\|_{4} .
$$

We now deduce a higher order estimate for the error in $H^{2}$.

THEOREM 3.3. If $h_{\Delta}$ is sufficiently small, then

$$
\left\|u-u_{\Delta}\right\|_{2} \leqslant C h_{\Delta}\left\|u-u_{\Delta}\right\|_{3} .
$$

If also $u \in H^{4}$, then

$$
\left\|u-u_{\Delta}\right\|_{2} \leqslant C h_{\Delta}^{2}\|u\|_{4} .
$$

Proof. Since the Dirichlet problem for $A$ is well-posed, we have the a priori estimate

$$
\left\|u-u_{\Delta}\right\|_{2} \leqslant C\left\|A\left(u-u_{\Delta}\right)\right\|_{0} .
$$

Hence the theorem follows from the following lemma and Corollary 3.2.

LEMMA 3.4. If $h_{\Delta}$ is sufficiently small, then

$$
\left\|A\left(u-u_{\Delta}\right)\right\|_{0} \leqslant C h_{\Delta}\left\|u-u_{\Delta}\right\|_{3} .
$$

Proof. Define $w \in \tilde{H}^{3}$ by $w^{(4)}=A\left(u-u_{\Delta}\right)$. Then $\|w\|_{4} \leqslant C\left\|A\left(u-u_{\Delta}\right)\right\|_{0}$ and

$$
\begin{aligned}
\left\|A\left(u-u_{\Delta}\right)\right\|_{0}^{2} & =B\left(u-u_{\Delta}, w\right)=\inf _{v \in \tilde{M}_{3}(\Delta)} B\left(u-u_{\Delta}, w-v\right) \\
& \leqslant C\left\|u-u_{\Delta}\right\|_{3} \inf _{v \in \tilde{M}_{3}(\Delta)}\|w-v\|_{3} \leqslant C h_{\Delta}\left\|u-u_{\Delta}\right\|_{3}\|w\|_{4} \\
& \leqslant C h_{\Delta}\left\|u-u_{\Delta}\right\|_{3}\left\|A\left(u-u_{\Delta}\right)\right\|_{0} .
\end{aligned}
$$


Remark. If $k<2$, then it is not true that

$$
\left\|u-u_{\Delta}\right\|_{k} \leqslant C h_{\Delta}^{4-k}\|u\|_{4},
$$

as the following argument [23] shows for $k=1$. Consider the simple case $a \equiv 1$, $b \equiv c \equiv 0$, and let $u$ be any $H^{5}$ function that $u^{(4)}(0)=u^{(4)}(1)=0$ and for which $u^{(4)}$ does not vanish identically. Then

$$
\begin{aligned}
\left\|u^{\prime \prime \prime}-u_{\Delta}^{\prime \prime}\right\|_{0}^{2} & =B\left(u-u_{\Delta}, u-u_{\Delta}\right)=B\left(u-u_{\Delta}, u\right) \\
& =-\left(u^{\prime \prime}-u_{\Delta}^{\prime \prime}, u^{(4)}\right)=\left(u^{\prime}-u_{\Delta}^{\prime}, u^{(5)}\right) .
\end{aligned}
$$

Hence if (3.12) holds for $k=1$, then there exists a constant $C$ depending on $u$ but independent of the mesh $\Delta$ such that

$$
\left\|u^{\prime \prime \prime}-u_{\Delta}^{\prime \prime \prime}\right\|_{0} \leqslant C h_{\Delta}^{3 / 2}
$$

But (3.13) is impossible since $u_{\Delta}^{\prime \prime \prime}$ is piecewise constant and $u^{\prime \prime \prime}$ is not constant.

By contrast, if a standard Galerkin method is used to define $u_{\Delta} \in \tilde{M}_{3}(\Delta)$, then (3.12) can easily be shown to hold for $-2 \leqslant k \leqslant 1$. If the mesh is restricted to a quasiuniform family an inverse property can be used to establish (3.11) for the Galerkin method with $k=2$ and 3 as well.

It has long been known [21] that nodal collocation of second order differential equations by cubic splines does not afford optimal order estimates for the solution values (at least in $L^{\infty}$ ). Archer [9], [10] and Daniel and Swartz [35] have shown in the case of uniform mesh spacing how to perturb the collocation equations to correct this defect.

Appendix.

A.1. Construction of $\theta(s)$ in (2.3.5) [81]. Let (2.3.6) be satisfied and let $U(s):=A_{0}^{-1}(s) C_{0}(s, s)$. Then let

$$
V(s):=\left\{(I+U(s))^{1 / 2}(I-U(s))^{1 / 2}\right\}^{-1},
$$

where the square roots are defined by means of the Dunford-Taylor integral with eigenvalues having positive real parts [57, I.5.6]. With $V(s)$ define

$$
N_{+}(s):=V(S)(I+U(s)) \text {. }
$$

Then the matrix function

$$
H(s):=\int_{0}^{\infty} \exp \left(-N_{+}^{*}(s) \tau\right) \exp \left(-N_{+}(s) \tau\right) d \tau
$$

can be defined $[19,10.18]$, and

$$
\theta(s)=(-i)^{m} H(s) V(s) A_{0}^{-1}(s)
$$

gives the desired matrix in (2.3.5).

A.2. One of the Singular Integral Equations of the Direct Method in Plane Elasticity. The basic relation for the direct method in plane isotropic homogeneous elasticity is given by Betti's formula

$$
\varepsilon u(z)=\int_{\Gamma}\left\{\frac{1}{2} S(z, \zeta) u(\zeta)-G(z, \zeta) t(\zeta)\right\} d s_{\zeta}-\frac{1}{2}\left(\begin{array}{c}
\omega_{1} \\
\omega_{2}
\end{array}\right)-\frac{1}{2} \omega_{3}\left(\begin{array}{c}
y \\
-x
\end{array}\right)
$$


with $\varepsilon=1$ for interior problems and $\varepsilon=-1$ for exterior problems. $u=\left(u_{1}, u_{2}\right)$ denotes the vector of displacement, and

$$
t(z)=\left(\begin{array}{l}
\left((\lambda+2 \mu) \frac{\partial u_{1}}{\partial x}+\lambda \frac{\partial u_{2}}{\partial y}\right) \frac{d y}{d s}-\mu\left(\frac{\partial u_{1}}{\partial y}+\frac{\partial u_{2}}{\partial x}\right) \frac{d x}{d s} \\
\mu\left(\frac{\partial u_{1}}{\partial y}+\frac{\partial u_{2}}{\partial x}\right) \frac{d y}{d s}-\left((\lambda+2 \mu) \frac{\partial u_{2}}{\partial y}+\lambda \frac{\partial u_{1}}{\partial x}\right) \frac{d y}{d s}
\end{array}\right)
$$

denotes the traction on the boundary curve $\Gamma$. $S$ denotes the traction of point loads in (2.3.7), and $G$ is the fundamental solution of the elasticity equations

$$
\mu \Delta u+(\lambda+\mu) \operatorname{grad} \operatorname{div} u=0,
$$

namely

$$
\begin{aligned}
G_{j k} & =-\frac{1}{2 \pi \mu} \log |z-\zeta| \delta_{j k}+\frac{\lambda+\mu}{8 \pi \mu(2 \mu+\lambda)} \frac{\partial^{2}}{\partial z_{j} \partial z_{k}}\left(|z-\zeta|^{2} \log |z-\zeta|\right) \\
& =\frac{\lambda+3 \mu}{4 \mu(2 \mu+\lambda)}\left\{-\frac{1}{\pi} \log |z-\zeta| \delta_{j k}+L\right\}
\end{aligned}
$$

with $L$ given by (2.3.13) $\left(z=z_{1}+i z_{2}=x+i y\right)$. From (A.2.1) one finds Somigliana's identity on the boundary, i.e., for interior problems with $\varepsilon=1$ and $\omega=0$ and for exterior problems $\varepsilon=-1$ and $\omega$ describing the rigid motion,

$$
\begin{aligned}
\varepsilon u(z)= & 2 \int_{\Gamma}\left\{\frac{1}{2} S(z, \zeta) u(\zeta)-G(z, \zeta) t(\zeta)\right\} d s_{\zeta} \\
& -\left(\begin{array}{c}
\omega_{1} \\
\omega_{2}
\end{array}\right)-\omega_{3}\left(\begin{array}{c}
y \\
-x
\end{array}\right), \quad z \in \Gamma .
\end{aligned}
$$

(For this formulation see also [4], [62].)

The first fundamental problem. Here the displacement on the boundary is given,

$$
\left.u\right|_{\Gamma}=\left(\varphi_{1}, \varphi_{2}\right)^{T}
$$

Inserting $\left.u\right|_{\Gamma}$ into (A.2.2) gives the integral equation

$$
\begin{aligned}
\int_{\Gamma}\left\{-\frac{1}{\pi}\right. & \log |z-\zeta| I+L(z, \zeta)\} t(\zeta) d s_{\zeta} \\
& =\frac{2 \mu(\lambda+2 \mu)}{\lambda+3 \mu}\left\{\varepsilon \varphi(z)-\int_{\Gamma} S(z, \zeta) \varphi(\zeta) d s_{\zeta}\right\}+\left(\begin{array}{ccc}
1 & 0 & y \\
0 & 1 & -x
\end{array}\right) \tilde{\omega}
\end{aligned}
$$

for the unknown boundary traction $t$, where for the interior problem $\tilde{\omega}=0$. For the equilibrium state one further has

$$
\int_{\Gamma} t d s=0 \text { and } \int_{\Gamma}\left(t_{1} y-t_{2} x\right) d s=0
$$

(A.2.3) and (A.2.4) is just the form (2.3.12), (2.3.13) providing the unique solution $t$ and $\tilde{\omega}=-(2 \mu(\lambda+2 \mu) /(\lambda+3 \mu)) \omega[51]$.

The second fundamental problem. For the second problem on $\Gamma$ the tractions are given,

$$
\left.t\right|_{\Gamma}=\left(\psi_{1}, \psi_{2}\right)^{T}
$$


satisfying the equilibrium conditions

$$
\int_{\Gamma} \psi d s=0 \text { and } \int_{\Gamma}\left(\psi_{2} x-\psi_{1} y\right) d s=0
$$

Inserting $\left.t\right|_{\Gamma}$ into (A.2.2) gives the singular integral equation

$$
(\mathrm{A} .2 .5) \quad \varepsilon u(z)+\int_{\Gamma} S(z, \zeta) u(\zeta) d s_{\zeta}+\left(\begin{array}{ccc}
1 & 0 & y \\
0 & 1 & -x
\end{array}\right) \omega=2 \int_{\Gamma} G(z, \zeta) \psi d s_{\zeta}
$$

for the yet unknown displacement. For $\varepsilon=1$ the rigid motions

$$
u_{0}(z)=\left(a_{1}, a_{2}\right)^{T}+\gamma(y,-x)^{T}, \quad a_{1}, a_{2}, \gamma \in \mathbf{R},
$$

however, define eigensolutions of (A.2.5). Therefore we modify (A.2.5) in the form (2.3.7) which determines $u$ uniquely in both cases $\varepsilon= \pm 1$.

Note Added in Proof. In response to the prepublication appearance of this paper $\mathrm{J}$. Elschner and G. Schmidt have furnished in [106] a proof of the approximation property (2.1.4) and the inverse property (2.1.30) in the general case stated above (see the discussion following these two equations). In the same work they question the generality of the estimate $\left|J u-J_{\Delta} u\right| \leqslant C h_{\Delta}^{s}, s \in(1 / 2,2]$, used in the proofs of Theorems 2.1.4 and 2.1.6. This estimate is indeed valid (with the constant $C$ depending on $s$ but independent of $\Delta$ ). It follows from the stronger result $\left\|u-I_{\Delta} u\right\|_{0}$ $\leqslant C h_{\Delta}^{s}\|u\|_{s}$ where $I_{\Delta} u$ is the piecewise linear interpolant of $u$. To establish this latter estimate we note that for $s \in\{1,2\}$ it is clear, and hence also for $s \in[1,2]$ by interpolation, so it suffices to consider $s \in(1 / 2,1)$. Now for $a<b, f \in C^{1}([a, b])$, let $I f$ be the linear function interpolating $f$ on $\{a, b\}$. Then

$$
\int_{a}^{h}|f(x)-I f(x)|^{2} d x \leqslant C(b-a)^{2 s} \int_{a}^{b} \int_{a}^{b} \frac{|f(x)-f(y)|^{2}}{|x-y|^{1+2 s}} d x d y,
$$

as follows easily from the Sobolev embedding theorem, the vanishing of the left-hand side for $f$ constant, and a trivial scaling argument. Applying this inequality with $a=x_{i-1}, b=x_{i}, f=u$, and adding over $i$, we get

$$
\left\|u-I_{\Delta} u\right\|_{0} \leqslant C h_{\Delta}^{2 s} \int_{0}^{1} \int_{0}^{1} \frac{|u(x)-u(y)|^{2}}{|x-y|^{1+2 s}} d x d y \leqslant C h_{\Delta}^{2 s}\|u\|_{s}^{2}, \quad u \in H^{s} \cap C^{1}([0,1]) \text {. }
$$

(The last inequality is well-known. A proof is written out in, e.g., [106].) Finally by density the equation holds for all $u \in H^{s}$.

\author{
Department of Mathematics and \\ Institute for Physical Science and Technology \\ University of Maryland \\ College Park, Maryland 20742 \\ Department of Mathematics \\ Technische Hochschule Darmstadt \\ Schlossgartenstr. 7 \\ D-6100 Darmstadt \\ Federal Republic of Germany
}

1. M. S. ABOU El-SEOUd, Numerische Behandlung von schwach singulären Integralgleichungen erster Art, Doctoral Dissertation, Technische Hochschule Darmstadt, Germany, 1979.

2. M. S. ABOU EL-SEOUd, “Kollokationsmethode für schwach singuläre Integralgleichungen erster Art,” Z. Angew. Math. Mech., v. 59, 1979, pp. T45-T47. 
3. M. S. AGRANOvich, "Elliptic singular integro-differential operators," Russian Math. Surveys. v. 20, 1965, pp. 1-122.

4. J. F. AHNER \& G. C. HSiAo, "On the two-dimensional exterior boundary-value problems of elasticity," SIAM J. Appl. Math., v. 31, 1976, pp. 677-685.

5. M. A. ALEKSIDZE, The Solution of Boundary Value Problems with the Method of the Expansion with Respect to Nonorthonormal Functions, "Nauka", Moscow, 1978. (Russian)

6. P. ANSElone, Collectively Compact Operator Theory, Prentice-Hall, London, 1971.

7. H. ANTES, Die Splineinterpolation zur Lösung von Integralgleichungen und ihre Anwendung bei der Berechnung von Spannungen in krummlinig berandeten Scheiben, Doctoral Dissertation R-W-TH Aachen, Germany, 1970.

8. H. ANTES, "Splinefunktionen bei der Lösung von Integralgleichungen," Numer. Math., v. 19, 1972, pp. 116-126.

9. D. ARcher, Some Collocation Methods for Differential Equations, Ph.D. Thesis, Rice University, Houston, 1973.

10. D. ARCHER, "An $O\left(h^{4}\right)$ cubic spline collocation method for quasilinear parabolic equations," SIAM J. Numer. Anal., v. 14, 1977, pp. 620-637.

11. D. W. ARThur, "The solution of Fredholm integral equations using spline functions," J. Inst. Math. Appl., v. 11, 1973, pp. 121-129.

12. K. E. AtKinson, $A$ Survey of Numerical Methods for the Solution of Fredholm Integral Equations of the Second Kind, SIAM, Philadelphia, Pa., 1976.

13. F. V. ATKINSON, “On relatively regular operators," Acta Sci. Math. Szeged., v. 15, 1953, pp. 38-56.

14. J. P. Aubin, Approximation of Elliptic Boundary Value Problems, Wiley, New York, 1972.

15. I. BABUSKA \& A. K. Azız, "Survey lectures on the mathematical foundations of the finite element method," in The Mathematical Foundation of the Finite Element Method with Applications to Partial Differential Equations (A. K. Aziz, ed.), Academic Press, New York, 1972, pp. 3-359.

16. I. BABUSKKA, "Error-bounds for finite element method," Numer. Math., v. 16, 1970, pp. 322-333.

17. C. BAKER, The Numerical Treatment of Integral Equations, Clarendon Press, Oxford, 1977.

18. P. K. Banerjee \& R. Butterfield (eds.), Developments in Boundary Element Methods, Appl. Science Publ., London, 1979.

19. R. Bellman, Introduction to Matrix Analysis, McGraw-Hill, New York, 1960.

20. L. Bolteus \& O. Tullberg, "BEMSTAT-A new type of boundary element program for two-dimensional elasticity problems", in Boundary Element Methods (C. A. Brebbia, ed.), Springer-Verlag, Berlin and New York, 1981, pp. 518-537.

21. C. DE BooR, The Method of Projections as Applied to the Numerical Solution of Two Point Boundary Value Problems Using Cubic Splines, Ph.D. Thesis, University of Michigan, Ann Arbor, Mich., 1966.

22. C. DE Boor \& B. Swartz, "Collocation at Gaussian points," SIAM J. Numer. Anal., v. 10, 1973, pp. 582-606.

23. J. H. Bramble \& R. Scott, "Simultaneous approximation in scales of Banach spaces," Math. Comp., v. 32, 1978, pp. 947-954.

24. C. A. BrebBia (ed.), The Boundary Element Method for Engineers, Pentech Press, London, Plymouth, 1978.

25. C. A. BrebBiA (ed.), New Developments in Boundary Element Methods, CML Publ., Southampton, 1980.

26. C. A. BrebBiA (ed.), Boundary Element Methods, Springer-Verlag, Berlin and New York, 1981.

27. C. A. Brebbia (ed.), Progress in Boundary Element Methods, vol. 1 ff., Pentech Press, London, Plymouth, 1981.

28. G. BRUHN \& W. WeNDlAND, "Über die näherungsweise Lösung von linearen Funktionalgleichungen," in Funktionalanalysis, Approximationstheorie, Numerische Mathematik (L. Collatz, ed.), Internat. Ser. Numer. Math., v. 7, Birkhäuser, Basel, 1967, pp. 136-144.

29. J. CEA, "Approximation variationelle des problèmes aux limites," Ann. Inst. Fourier (Grenoble), v. 14, 1964, pp. $345-444$.

30. G. A. Chander, Superconvergence of Numerical Solutions to Second Kind Integral Equations, Ph.D. Thesis, Australian National University, 1979.

31. S. Christiansen, "Condition number of matrices derived from two classes of integral equations," Math. Methods Appl. Sci., v. 3, 1981, pp. 364-392.

32. S. Christiansen \& E. B. HANSEN, "A direct integral equation method for computing the hoop stress in plane isotropic sheets," J. Elasticity, v. 5, 1975, pp. 1-14.

33. L. Collatz, The Numerical Treatment of Differential Equations, Springer-Verlag, Berlin and New York, 1966. 
34. T. A. CRUSE, "Application of the boundary-integral equation solution method in solid mechanics," in Variational Methods in Engineering, Dept. Civil Eng., Southampton Univ., England, 1972, pp. 9.1-9.29.

35. J. W. Daniel \& B. K. Swartz, "Extrapolated collocation for two-point boundary value problems using cubic splines," J. Inst. Math. Appl., v. 16, 1975, pp. 161-174.

36. J. C. DiAz, “A collocation-Galerkin method for two-point boundary value problems using piecewise polynomial spaces," SIAM J. Numer. Anal., v. 14, 1977, pp. 844-859.

37. G. FICHERA, "Linear elliptic equations of higher order in two independent variables and singular integral equations," in Proc. Conf. Partial Differential Equations and Cont. Mechanics, Univ. of Wisconsin Press, 1961, pp. 55-80.

38. P. J. T. FilipPI, “Layer potentials and acoustic diffraction," J. Sound Vibration, v. 54, 1977, pp. 473-500.

39. G. J. Fix \& G. Strang, An Analysis of the Finite Element Method, Prentice-Hall, Englewood Cliffs, N.J., 1973.

40. D. GAIER, “Intergralgleichungen erster Art und konforme Abbildung," Math. Z., v. 147, 1976, pp. $113-129$.

41. J. Giroire \& J. C. NedEleC, “Numerical solution of an exterior Neumann problem using a double layer potential,” Math. Comp., v. 32, 1978, pp. 973-990.

42. G. HAMmerlin \& L. L. Schumaker, "Procedures for kernel approximation and solution of Fredholm integral equations of the second kind," Numer. Math., v. 34, 1980, pp. 125-141.

43. E. B. HANSEN, "Numerical solution of integro-differential and singular equations for plate bending problems,” J. Elasticity, v. 6, 1976, pp. 39-56.

44. E. B. HANSEN, "An integral equation method for stress concentration problems in cylindrical shells,” J. Elasticity, v. 7, 1977, pp. 283-305.

45. J. K. Hayes, D. K. Kahaner \& R. G. Kellner, “An improved method for numerical conformal mapping," Math. Comp., v. 26, 1972, pp. 327-334.

46. H. P. Helfrich, "Simultaneous approximation in negative norms of arbitrary order," RAIRO Numer. Anal., v. 15, 1981, pp. 231-235.

47. St. Hildebrandt \& E. Wienholtz, "Constructive proofs of representation theorems in separable Hilbert space," Comm. Pure Appl. Math., v. 17, 1964, pp. 369-373.

48. H.-P. HoIDN, Die Kollokationsmethode angewandt auf die Symmsche Integralgleichung, Doctoral Dissertation, ETH Zürich, Switzerland, 1983.

49. E. N. Houstis, “A collocation method for systems of nonlinear ordinary differential equations," $J$. Math. Anal. Appl., v. 62, 1978, pp. 24-37.

50. G. C. HsiaO, P. Kopp \& W. L. Wendland, “A Galerkin collocation method for some integral equations of the first kind," Computing, v. 25, 1980, pp. 89-130.

51. (j. C. HSiaO, P. KopP \& W. L. WendLAND, "Some applications of a Galerkin-collocation method for integral equations of the first kind." (To appear.) Preprint 768, Fachbereich Mathematik, Technische Hochschule Darmstadt, Germany, 1983.

52. G. C. Hsiao \& R. C. MACCAMY, "Solution of boundary value problems by integral equations of the first kind," SIAM Rev., v. 15, 1973, pp. 687-705.

53. G. C. HSIAO \& W. L. Wendland, “A finite element method for some integral equations of the first kind," J. Math. Anal. Appl., v. 58, 1977, pp. 449-481.

54. G. C. Hsiao \& W. L. Wendland, "The Aubin-Nitsche lemma for integral equations," J. Integral Equations, v. 3, 1981, pp. 299-315.

55. G. C. Hsiao \& W. L. Wendland, "Super approximation for boundary integral methods," in Advances in Computer Methods for Partial Differential Equations-IV (R. Vichnevetsky \& R. S. Stepleman, eds.), IMACS Symp., Dept. of Comput. Sci., Rutgers Univ., New Brunswick, N.J., 1981, pp. 200-205

56. M. A. Jaswon \& G. T. Symm, Integral Equation Methods in Potential Theory and Elastostatics, Academic Press, London, 1977.

57. T. KATO, Perturbation Theory for Linear Operators, Springer-Verlag, Berlin and New York, 1966.

58. J. J. Kohn \& L. Nirenberg, "On the algebra of pseudo-differential operators," Comm. Pure. Appl. Math., v. 18, 1965, pp. 269-305.

59. A. KRAwIETZ, Energetische Behandlung des Singularitätenverfahrens, Doctoral Dissertation, Technical University, Berlin, Germany, 1972.

60. G. Kuhn \& W. MöhrmanN, "Boundary element methods in elastostatics: Theory and applications," Appl. Math. Modelling, v. 7, 1983, pp. 97-105.

61. V. D. KUPRADZE, Randwertaufgaben der Schwingungstheorie und Integralgleichungen, Deutscher Verlag d. Wiss., Berlin, 1956. 
62. V. D. Kupradze, Potential Methods in the Theory of Elasticity, Israel Program for Scientific Transl., Jerusalem, 1965.

63. V. D. Kupradze, T. G. Gegelia, M. O. Basheleishvili \& T. V. Burchuladze, Three-Dimensional Problems of the Mathematical Theory of Elasticity and Thermoelasticity, North-Holland, Amsterdam, 1979.

64. U. Lamp, T. Schleicher, E. Stephan \& W. L. Wendland, "The boundary integral method for a plane mixed boundary value problem," in Advances in Computer Methods for Partial Differential Equations-IV (R. Vichnevetsky \& R. S. Stepleman, eds.), IMACS Symp., Dept. of Comput. Sci., Rutgers Univ., New Brunswick, N.J., 1981, pp. 222-229.

65. U. Lamp, T. Schleicher, E. Stephan \& W. L. Wendland, "Galerkin collocation for an improved boundary element method for a plane mixed boundary value problem," Computing. (To appear.)

66. T. R. LuCAS \& G. W. ReDdien, "Some collocation methods for nonlinear boundary value problems," SIAM J. Numer. Anal., v. 9, 1972, pp. 341-356.

67. E. Martensen, "Berechnung der Druckverteilung an Gitterprofilen in ebener Potentialströmung mit einer Fredholmschen Integralgleichung," Arch Rational Mech. Anal., v. 3, 1959, pp. 235-270.

68. G. MeHLhoRN, Ein Beitrag zum Kipp-Problem bei Stahlheton- und Spannhetonträgern, Doctoral Dissertation, D17, Technische Hochschule Darmstadt, Germany, 1970.

69. S. G. Michlin \& S. PRössDORF, Singuläre Integraloperatoren, Akademie-Verlag, Berlin, 1980.

70. N. I. Muskhelishvili, Some Basic Problems of the Mathematical Theory of Elasticity, Noordhoff, Groningen, 1963.

71. N. I. MUSKHELISHVILI, Singular Integral Equations, Noordhoff, Groningen, 1953.

72. G. G. Mustoe \& I. C. Mathews, "Direct boundary integral methods, point collocation and variational procedures." (To appear.)

73. J. C. NeDelec, Approximation des Equations Intégrales en Mecanique et en Physique, Lecture Notes, Centre de Mathématiques Appliquées, Ecole Polytechnique, Palaiseau, France, 1977.

74. J. A. Nitsche, "Zur Konvergenz von Näherungsverfahren bezüglich verschiedener Normen," Numer. Math., v. 15, 1970, pp. 224-228.

75. Ben NoBle, A Bibliography on: "Methods for solving integral equations," Math. Res. Center Tech. Report 1176 and 1177, Madison, Wisc., 1971.

76. F. A. Oliveira, "Collocation and residual correction," Numer. Math., v. 36, 1980, pp. 27-31.

77. R. S. Palais, Seminar on the Atiyah-Singer Index Theorem, Princeton Univ. Press, Princeton, N.J., 1965.

78. P. M. Prenter, "A collocation method for the numerical solution of integral equations," SIAM J. Numer. Anal., v. 10, 1973, pp. 570-581.

79. S. Prössdorf \& B. Silbermann, Projektionsverfahren und die näherungsweise Lösung singulürer Gleichungen, Teubner, Leipzig, 1977.

80. S. PrösSDORF \& G. SCHMidT, "A finite element collocation method for singular integral equations," Math. Nachr., v. 100, 1981, pp. 33-60.

81. S. PRössDorf \& G. SCHMIDT, "A finite element collocation method for systems of singular integral equations," Preprint P-MATH-26/81, Akademie der Wissenschaften der DDR, Institut für Mathematik, DDR-1080 Berlin, Mohrenstr. 39, 1981.

82. G. R. RICHTER, "Superconvergence of piecewise polynomial Galerkin approximations for Fredholm integral equations of the second kind," Numer. Math., v. 31, 1978, pp. 63-70.

83. F. Riesz \& Bela Sz.-NaGY, Vorlesungen über Funktionalanalysis, Deutscher Verlag d. Wiss., Berlin, 1956.

84. F. J. Rizzo, "An integral equation approach to boundary value problems of classical elastostatics," Quart. Appl. Math., v. 25, 1967, pp. 83-95.

85. M. N. Le Roux, Resolution Numérique du Probleme du Potential dans le Plan par une Méthode Variationelle d'Eléments Finis, Doctoral thesis, Université de Rennes, Sér. A No. 347 ser. 38, France, 1974.

86. M. N. LE Roux, "Équations intégrales pour le problème du potential électrique dans le plan," $C . R$. Acad. Sci. Paris, Ser. A, 1974, p. 278.

87. R. D. Russell, "Collocation for systems of boundary value problems," Numer. Math., v. 23, 1974, pp. 119-133.

88. E. SCHAFER, "Fehlerabschätzungen für Eigenwertnäherungen nach der Ersatzkernmethode bei Integralgleichungen," Numer. Math., v. 32, 1979, pp. 281-290.

89. L. L. SChUMAKER, Spline Functions: Basic Theory, Wiley, New York, 1981.

90. R. SeEley, "Topics in pseudo-differential operators," in Pseudo-Differential Operators (L. Nirenberg, ed.), CIME, Cremonese, Roma, 1969, pp. 169-305.

91. R. SHAw ET AL., Innovative Numerical Analysis for the Engineering Sciences, The University Press of Virginia, 1980. 
92. E. Stephan \& W. L. Wendland, Remarks to Galerkin and Least Squares Methods with Finite Elements for General Elliptic Problems Lecture Notes in Math., v. 564, Springer-Verlag, Berlin, 1976, pp. 461-471; Manuscripta Geodaetica, v. 1, 1976, pp. 93-123.

93. F. Stummel, Rand- und Eigenweitaufgaben in Sobolevschen Räumen, Lecture Notes in Math., vol. 102, Springer-Verlag, Berlin, 1969.

94. B. Swartz \& B. WendrofF, "The relation between the Galerkin and collocation methods using smooth splines," SIAM J. Numer. Anal., v. 11, 1974, pp. 994-996.

95. G. T. Syмm, “Numerical mapping of exterior domains,” Numer. Math., v. 10, 1967, pp. 437-445.

96. I. SzABO, Höhere Technische Mechanik, Springer-Verlag, Berlin, 1956.

97. F. Treves, Introduction to Pseudodifferential and Fourier Integral Operators I, Plenum Press, New York, 1980.

98. V. V. Voronin \& V. A. Сесоно, "An interpolation method for solving an integral equation of the first kind with a logarithmic singularity," Soviet Math. Dokl., v. 15, 1974, pp. 949-952.

99. J. WATSON, "Hermitian cubic boundary elements for plane problems of fracture mechanics," Res. Mechanica, 1981.

100. W. L. WENDLAND, “On Galerkin collocation methods for integral equations of elliptic boundary value problems," in Numerical Treatment of Integral Equations (J. Albrecht \& L. Collatz, eds.), Internat. Ser. Numer. Math., vol. 53, Birkhäuser, Basel, 1980, pp. 244-275.

101. W. L. WENDLAND, "On the asymptotic convergence of boundary integral methods," in Boundary Element Methods (C. A. Brebbia, ed.), Springer-Verlag, Berlin and New York, 1981, pp. 412-430.

102. W. L. WENDLAND, "Asymptotic convergence of boundary element methods," in Lectures on the Numerical Solution of Partial Differential Equations (I. Babuška, T.-P. Liu \& J. Osborn, eds.), Lecture Notes, vol. 20, Univ. of Maryland, College Park, MD, 1981, pp. 435-528.

103. W. L. Wendland, "Asymptotic accuracy and convergence," in Progress in Boundary Element Methods (C. A. Brebbia, ed.), Pentech Press, London, Plymouth, vol. 1, 1981, pp. 289-313.

104. H. Werner \& R. Schaback, Praktische Mathematik, Vol. II, Springer-Verlag, Berlin and New York, 1972.

105. O. S. ZiEnKIEwicz, The Finite Element Method, McGraw-Hill, London, 1977.

106. J. Elschner \& G. SChmidT, "On spline interpolation in periodic Sobolev spaces," Preprint P-MATH-01/83, Akademie der Wissenschaften der DDR, Institut für Mathematik, DDR 1080 Berlin, Mohrenstr. 39, 1983 (added in proof). 